US Army Corps

Engineer Research and

Development Center

Coastal Inlets Research Program

\title{
An Optimized Combined Wave and Current Bottom Boundary Layer Model for Arbitrary Bed Roughness
}

Richard Styles, Scott M. Glenn, and Mitchell E. Brown

June 2017

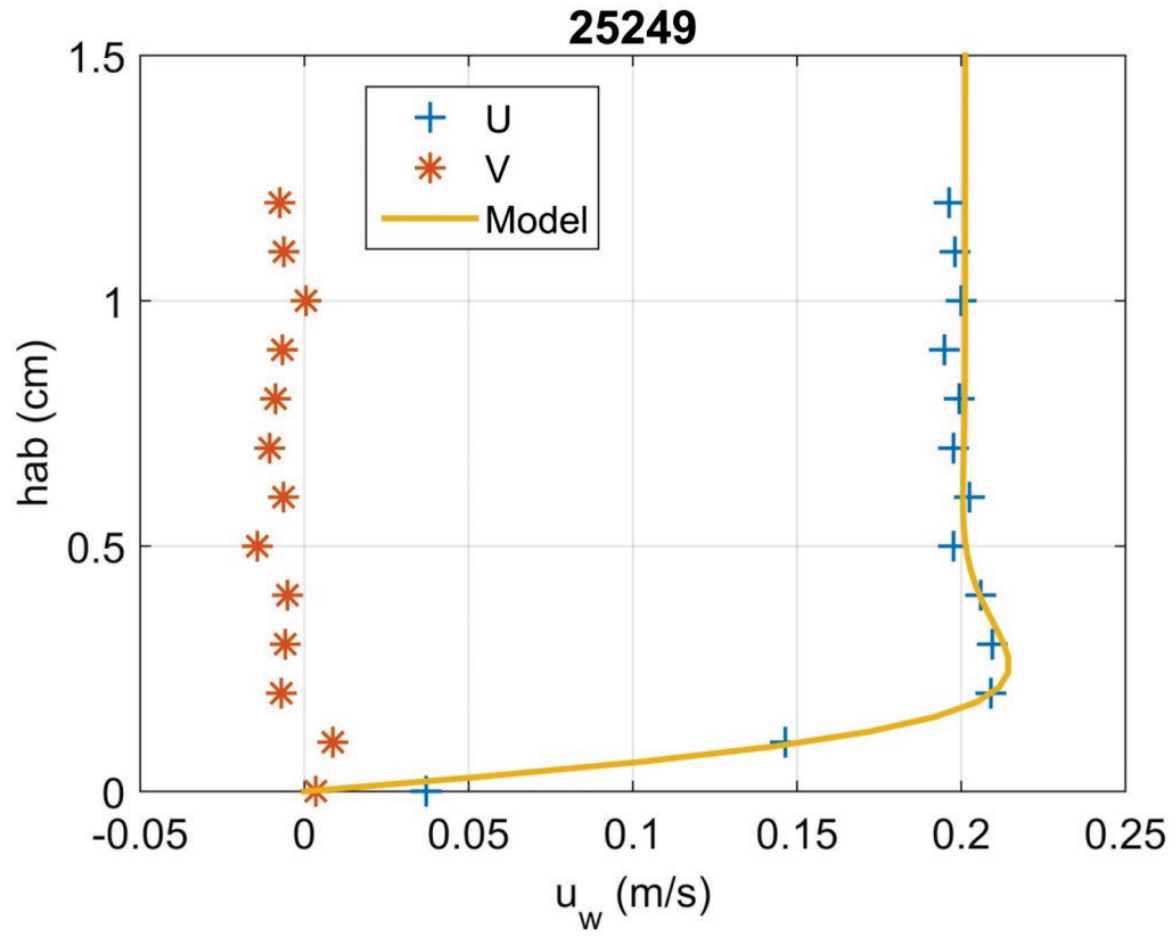


The U.S. Army Engineer Research and Development Center (ERDC) solves the nation's toughest engineering and environmental challenges. ERDC develops innovative solutions in civil and military engineering, geospatial sciences, water resources, and environmental sciences for the Army, the Department of Defense, civilian agencies, and our nation's public good. Find out more at www.erdc.usace.army.mil.

To search for other technical reports published by ERDC, visit the ERDC online library at http://acwc.sdp.sirsi.net/client/default. 


\section{An Optimized Combined Wave and Current Bottom Boundary Layer Model for Arbitrary Bed Roughness}

Richard Styles and Mitchell E. Brown

Coastal and Hydraulics Laboratory

U.S. Army Engineer Research and Development Center

3909 Halls Ferry Road

Vicksburg, MS 39180-6199

Scott M. Glenn

Institute of Marine and Coastal Sciences

Rutgers, The State University of New Jersey

57 U.S. Highway 1

New Brunswick, NJ 08901-8554

Final report

Approved for public release; distribution is unlimited.

Prepared for U.S. Army Corps of Engineers

Washington, DC 20314-1000

Under Project 462583, "Inlet Engineering Toolbox" 


\section{Abstract}

A robust method for computing the bed shear stress in unstratified combined wave and current flows is presented. The present approach follows from existing theories describing the nonlinear wave and current interaction in the benthic boundary layer but is designed for arbitrary wave, current, and roughness conditions, including the limiting case of pure waves or pure currents. The stress model is intended as a stand-alone application or for coupling to three-dimensional shelf circulation models, where a broad range of flow conditions are encountered. High-quality data for combined flows and pure waves are used with the present stress formulation to better refine empirical model closure constants in the fully rough turbulent regime. Introducing a first-order correction to the definition of the wave boundary layer thickness produces accurate estimates of both the measured friction factor and wave boundary layer height. A speed of convergence test indicates that the present model is more efficient than previous models that use the same turbulent closure scheme. This is primarily due to an improved solution algorithm that avoids the nested iterations common to established combined wave and current bottom boundary layer models.

DISCLAIMER: The contents of this report are not to be used for advertising, publication, or promotional purposes. Citation of trade names does not constitute an official endorsement or approval of the use of such commercial products. All product names and trademarks cited are the property of their respective owners. The findings of this report are not to be construed as an official Department of the Army position unless so designated by other authorized documents. 


\section{Contents}

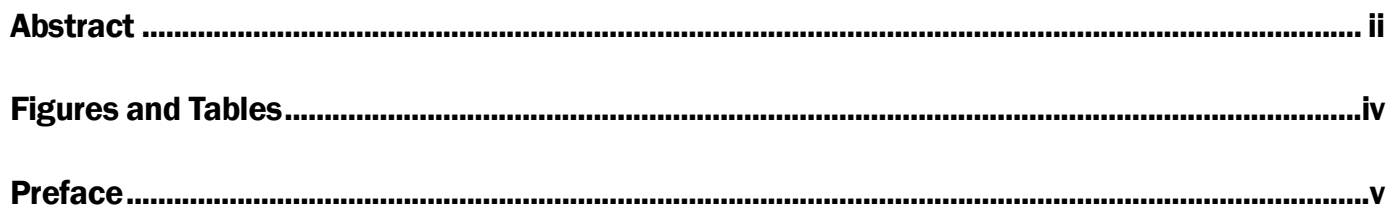

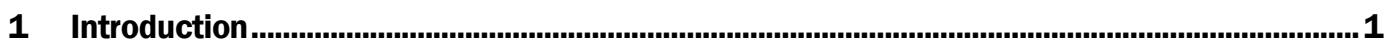

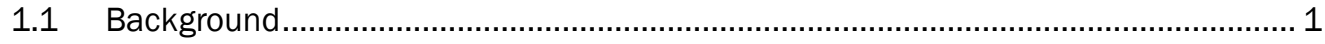

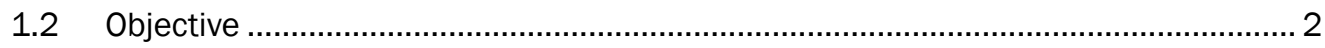

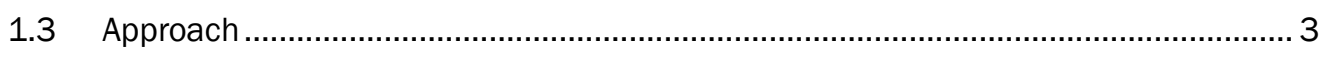

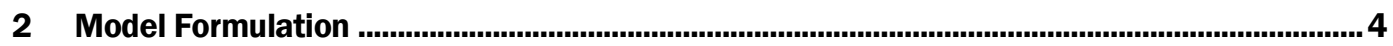

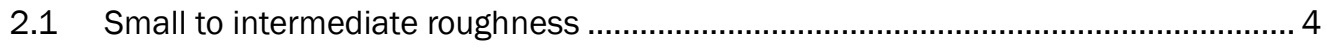

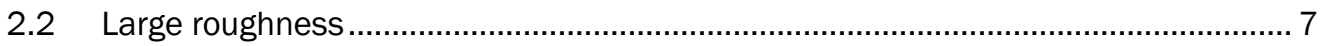

2.3 Large roughness with vanishingly small waves .................................................... 7

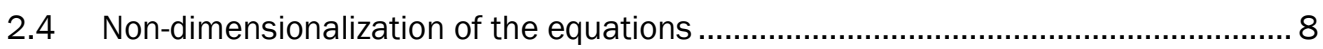

2.5 Solution algorithm for the three-layer model ........................................................ 11

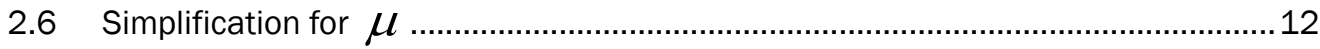

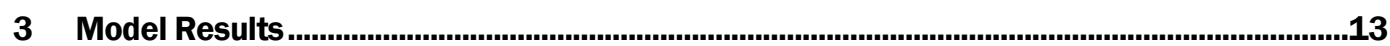

3.1 Fundamental model characteristics ................................................................ 13

3.2 Sensitivity to the direction between the wave and current .................................... 16

3.3 Bottom stress sensitivity to the eddy viscosity profile............................................. 18

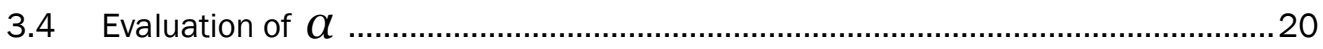

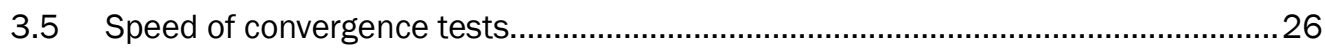

4 Summary

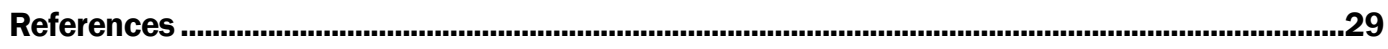

Appendix A: Derivation of Non-dimensional Wave Shear under Strong Waves............................32

Appendix B - Derivation of Non-dimensional Wave Shear under Weak Waves .............................35

Report Documentation Page 


\section{Figures and Tables}

\section{Figures}

Figure 1. Schematic illustrating eddy viscosity profiles. (a) The roughness is less than $z_{1}$ producing the three-layer continuous profile; (b) the roughness is greater than $z_{1}$ but less than $z_{2}$, so the eddy viscosity is constant near the bed; (c) the roughness length is large compared to the wave boundary layer height and increases linearly, indicative of a pure current boundary layer; (d) the eddy viscosity for a pure wave with a roughness less than $z_{1}$; (e) the roughness is greater than $z_{1}$ for a pure wave producing a constant eddy viscosity; and (f) the original GM discontinuous eddy viscosity profile.

Figure 2. Selected internal model parameters as a function of $z_{r} / z_{0}$ and $A_{b} / z_{0}$. Definitions of the internal variables are provided in the text. Values for $A_{b} / z_{0}$ range from $10^{-3}$ to $10^{6}$ in decadal increments.

Figure 3. Similar to Figure 2 but showing only $\mu, \epsilon$, and $\sigma$. Left column is for $u_{b} / u_{r}=10$ (large waves), and right column is for $u_{b} / u_{r}=0.1$ (large currents)

Figure 4. Sensitivity of the parameters $\epsilon$ and $\mu$ to the direction between the wave and the current.

Figure 5. Comparison of $\mu, \epsilon$, and $\sigma$ for the GM (thin solid), Styles and Glenn (2000) (dash), and two-layer continuous (thick solid) eddy viscosity profiles. $A_{b} / z_{0}$ ranges from $10^{-1}$ to $10^{3}$ in decadal increments.

Figure 6. Combined wave and current model calibration results for the closure parameters $\alpha$

Figure 7. Pure wave model calibration results for the closure parameters $\alpha$ and $\beta$

\section{Tables}

Table 1. Expressions for $\sigma$ derived from the current solution discussed in the text. Inequalities signify applicable ranges for a given expression.

Table 2. Speed of convergence tests comparing the present method with the unstratified version of the Styles and Glenn (2000) wave and current BBLM. For all model runs, $\phi_{c w}=0$ and $Z_{r}=100 \mathrm{~cm}$. The first three rows are for strong waves and currents (SS), the middle three rows are for strong waves and weak currents (SW), and the last three rows are for weak waves and strong currents (WS). The last two columns list normalized run-time (RT) and total number of iterations (N) for each method. The Styles and Glenn (2000) model uses a nested iteration scheme. The numbers in parentheses indicate the maximum number of iterations for the inner loop, which usually occurred during the first or second iteration of the outer loop. 


\section{Preface}

This study was conducted for Headquarters, U.S. Army Corps of Engineers (HQUSACE), Washington, DC, under the USACE Coastal Inlets Research Program (CIRP), Project 462583, "Inlet Engineering Toolbox." The HQUSACE CIRP Program Manager was Dr. Julie D. Rosati, U.S. Army Engineer Research and Development Center (ERDC), Coastal and Hydraulics Laboratory (CHL), Flood and Storm Protection Division (HF), Coastal Processes Branch (HF-C). Mr. Jeffrey A. McKee was the HQUSACE Navigation Business Line Manager overseeing the CIRP.

At the time of publication, Ms. Tanya Beck was Chief, HN-C, and Dr. Jackie Pettway was Chief, HN. Mr. W. Jeff Lillycrop (CHL) was the ERDC Technical Director for Civil Works and Navigation Research, Development, and Technology Transfer (RD\&T) portfolio. The Deputy Director of CHL was Mr. Jeffrey R. Eckstein, and the Director of CHL was Mr. José E. Sánchez.

The Commander of ERDC was COL Bryan S. Green, and the Director was Dr. David W. Pittman. 


\section{Introduction}

An important physical process for coastal circulation modeling is the interaction in the bottom boundary layer between waves and currents and how these both interact with the bottom to modify bedforms and move sediment. A very important result of wave-current interaction theorized over three decades ago is the enhanced current shear stress due to waves (Smith 1975; Grant and Madsen 1979). Repeated measurements on stormdominated shelves have illustrated that nonlinear wave-current interaction can significantly enhance the roughness of the bed and the stress felt by the current (e.g., Cacchione and Drake 1982; Wiberg and Smith 1983; Grant et al. 1984; Drake et al. 1992). Therefore, wave-current interaction is expected to play a dominant role in the momentum balance of low-frequency shelf motion and should be considered in any realistic modeling effort in stormdominated shelf regions. This is especially important if one of the primary purposes is to study shallow water sediment transport.

\subsection{Background}

Modeling studies of shelf circulation patterns that incorporate wave-current effects in the bottom boundary layer have been conducted in the past (e.g., Spaulding and Isaji 1985; Cooper and Thompson 1989; Signell et al. 1990; Davies and Lawrence 1994; Keen and Slingerland 1993a, b; Keen and Glenn 1994, 1995; Warner et al. 2008; Benetazzo et al. 2014). Keen and Glenn (1994) provide a brief summary of coupled and uncoupled versions of the Grant and Madsen (1979) (hereinafter referred to as GM) and Glenn and Grant (1987) bottom boundary layer models (BBLMs) implemented in shelf-circulation models. Their review identifies a number of responses directly related to enhanced bottom shear stress due to waves on continental shelves including a reduction in current speed near the bottom, modification of sediment transport rates, and enhanced turning of the current vector in the bottom Ekman layer that increases coastal upwelling and downwelling. Keen and Glenn (1995) also showed increased offshore rotation of the current vector during downwelling and reduction in bottom current speeds in shallow water in a simulation of storm and tidal flow in the Middle Atlantic Bight. Keen and Glenn (1998) carried out a quantitative skill assessment of model performance using moored current meter data from the Gulf of Mexico during Hurricane Andrew. One of the sensitivities they studied included a three-order of magnitude variation in bottom 
roughness length, the largest roughness serving as a surrogate for the enhanced apparent bottom roughness known to occur in combined wave and current flows. Modeled currents showed the greatest sensitivity to bed roughness when compared to bottom currents measured in a water depth of 15 meters $(\mathrm{m})$. Normalized peak speed differences between measured and modeled currents decreased when the apparent roughness was increased from 0.1 centimeter $(\mathrm{cm})$ to $10 \mathrm{~cm}$. Because their model did not include wave-current interaction, the roughness and stress fields could not evolve in response to changing wave conditions. Even so, the higher correlation between modeled and measured currents in shallow water for simulated roughness comparable to that associated with the presence of surface waves reemphasizes the fact that wave-current effects are important on stormdominated continental shelves. In addition to these earlier studies, there have been a wide variety of combined flow model applications in shallow water environments. However, development of new analytical models has not advanced significantly except for eddy viscosity formulations (Yuan and Madsen 2015; Styles and Bryant 2016). A general review of analytical combined wave and current models is provided by Nielsen (1992) and Styles and Bryant (2016) and references therein.

The above results are based on a streamlined version of the GM wave and current BBLM (Keen and Glenn 1994). Like the original GM model, the streamlined version assumes that the roughness length is small compared to the wave boundary layer height and that the height of the reference current needed to drive the model (usually the lowest grid point) is greater than the wave boundary layer height. For arbitrary roughness lengths and model grid heights, it is possible that under some conditions neither of these requirements will be met. The streamlined version also uses the discontinuous eddy viscosity adopted by GM and Glenn and Grant (1987), which has been shown to be less accurate than more physically reasonable continuous eddy viscosity profiles for combined flows (Glenn 1983; Madsen and Wikramanayake 1991; Lynch et al. 1997; Styles and Glenn 2000) and pure waves (Sleath 1991; Nielsen 1981, 1992; Davies and Villaret 1997). For this study, a new solution to the Styles and Glenn (2000) BBLM is derived that is more accurate than previous models referenced.

\subsection{Objective}

The objective of this technical report is to describe the theory and equations that accompany a MATLAB computer program. The model is an extension of the Styles and Glenn (2000) version of the GM model but has 
been modified to incorporate arbitrary roughness configurations and a broader range of turbulence closure schemes (i.e., different eddy viscosity profiles). A secondary objective is to conduct speed of convergence tests to gauge model performance relative to other widely-used bottom boundary layer models.

\subsection{Approach}

The approach adopted here is based on systematic scaling of the equations and careful selection of key non-dimensional parameters to produce a closed solution that is numerically stable. The algorithm is a stand-alone application that can be used to predict time-averaged bed shear stress, maximum bed shear stress for the wave, maximum combined bed shear stress for the wave and current, bottom roughness, and the current profile within the bottom boundary layer for combined wave and current flows. It is also used to compute the bottom stress and bed roughness in threedimensional shelf circulation models (Warner et al. 2008). The program also predicts sediment transport characteristics for non-cohesive beds including the reference concentration, concentration profile within the bottom boundary layer, and the depth-integrated concentration. The sediment transport algorithms can further be used to predict the concentration profile and total suspended load per grid cell.

In Chapter 2, the model formulation is described emphasizing the modifications required to extend the bottom stress theory to include very rough flow conditions. Model sensitivities to the eddy viscosity profile and a calibration of poorly constrained internal model closure constants is presented in Chapter 3. This is followed by a speed of convergence test, and the results are summarized in Chapter 4. 


\section{Model Formulation}

The stress model developed here follows that of GM, in which the maximum combined shear stress, $\tau_{c w}$, is written as the vector sum of the time-averaged component associated with the current, $\tau_{c}$, plus the maximum component associated with the wave, $\tau_{w m}$,

$$
\tau_{c w}=\tau_{c}+\tau_{w m}
$$

where boldface denotes a vector quantity. Writing the stresses in terms of their respective shear velocities, $u_{*}=(\tau / \rho)^{1 / 2}$, and taking the magnitude gives

$$
u_{*^{\prime} w}^{2}=\sqrt{u_{*_{c}}^{4}+2 u_{*_{c}}^{2} u_{* w m}^{2} \cos \phi_{c w}+u_{*_{w m}}^{4}},
$$

where $\rho$ is the fluid density and $\phi_{c w}\left(0 \leq \tau_{c w} \leq \pi / 2\right)$ is the angle between the wave and current. To obtain a closed set of equations, there is an adoption of the usual gradient transport relation for the wave,

$$
u_{* w v}^{2}=\lim _{z \rightarrow z_{0}}\left(K \frac{\partial u_{w}}{\partial z}\right)
$$

and for the current,

$$
u_{* c}^{2}=\lim _{z \rightarrow z_{0}}\left(K \frac{\partial U}{\partial z}\right)
$$

where $K$ is the time-independent eddy viscosity, $u_{w}$ is the modulus of the wave solution in the lower part of the wave boundary layer, $U$ is the magnitude of the horizontal current, $z$ is the vertical coordinate measured positive upwards from the bed, and $z_{0}$ is the hydraulic roughness. Given profiles for the eddy viscosity, wave, and current, the nonlinear system Equations (2-2), (2-3), and (2-4) can be solved to produce the bottom stress vectors $\tau_{c w}, \tau_{w m}$, and $\tau_{c}$.

\subsection{Small to intermediate roughness}

For conditions in which the height of the roughness elements are small in comparison to the wave boundary layer thickness, Glenn (1983) proposed 
the following three-layer continuous eddy viscosity over the original twolayer discontinuous formulation used by GM:

$$
\begin{aligned}
& K=\kappa u_{*} z \quad z>z_{2}, \\
& K=\kappa u_{*} z_{1} \quad z_{1}<z<z_{2}, \\
& K=\kappa u_{* c w} Z \quad z_{0}<z<z_{1},
\end{aligned}
$$

where $\kappa$ is von Karman's constant (0.4), $z_{1}$ is an arbitrary constant scale height, and $z_{2}=z_{1} u_{* c w} / u_{*_{c}}$, which is determined by matching the eddy viscosities at $Z=Z_{2}$ (Figure $1[\mathrm{a}]$ ).

Substituting Equation (2-5) into Equation (2-3) gives

$$
u_{* w m}^{2}=\kappa u_{* c w} z_{0} \frac{u_{b}}{l_{c w}} \Gamma_{w s}
$$

where the non-dimensional wave shear is defined by

$$
\left.\Gamma_{w s} \equiv \frac{l_{c w}}{u_{b}} \frac{\partial u_{w}}{\partial z}\right|_{z=z_{0}}=\left.\frac{1}{u_{b}} \frac{\partial u_{w}}{\partial \xi}\right|_{\xi=\xi_{0}}
$$

and $u_{b}$ is the bottom wave orbital velocity. The non-dimensional coordinate, $\xi=z / l_{c w}\left(\xi_{0}=z_{0} / l_{c w}\right)$, is originally derived from GM's governing equation for the wave, where the scale height of the wave boundary layer for combined flows, $l_{c w}$, is defined by

$$
l_{c w}=\kappa u_{*} c w / \omega
$$

and $\omega$ is the wave radian frequency. Since several eddy viscosities will be explored in this analysis, the non-dimensional wave shear is introduced as a convenience. The solution to $\Gamma_{w s}$ is provided in Styles and Glenn (2000). By virtue of the eddy viscosity, Equation (2-6) provides a relationship between $u_{*_{w m}}$ and $u_{*_{c m}}$. 
Figure 1. Schematic illustrating eddy viscosity profiles. (a) The roughness is less than $z_{1}$ producing the three-layer continuous profile; (b) the roughness is greater than $z_{1}$ but less than $z_{2}$, so the eddy viscosity is constant near the bed; (c) the roughness length is large compared

to the wave boundary layer height and increases linearly, indicative of a pure current boundary layer; (d) the eddy viscosity for a pure wave with a roughness less than $z_{1}$; (e) the roughness is greater than $z_{1}$ for a pure wave producing a constant eddy viscosity; and (f) the original GM discontinuous eddy viscosity profile.

3-layer eddy viscosity profile for the current
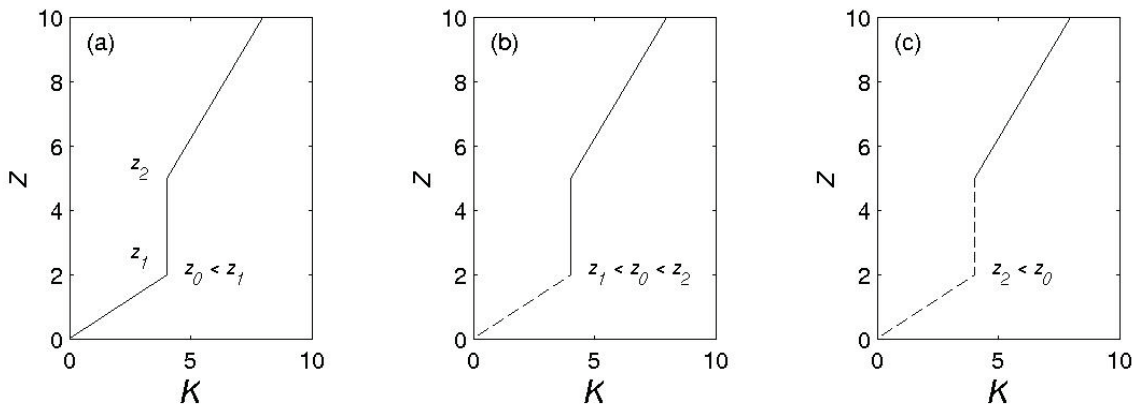

2-layer eddy viscosity profile for the wave

GM eddy viscosity profile
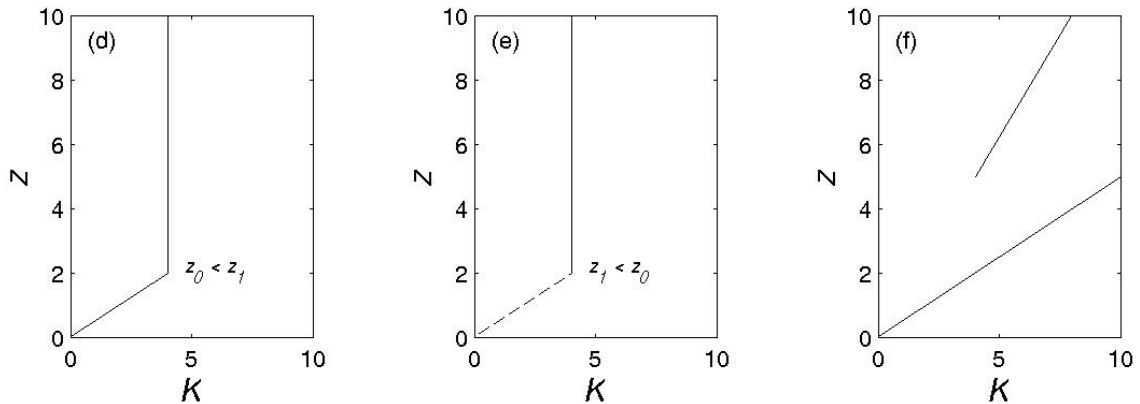

Substituting Equation (2-5) into Equation (2-4) and integrating gives the mean current profile:

$$
\begin{array}{ll}
U(z)=\frac{u_{*} c}{\kappa} \ln \left(\frac{z}{z_{2}}\right)+U\left(z_{2}\right) & z_{2}<z, \\
U(z)=\frac{u_{* c}^{2}}{\kappa u_{*} c w} \frac{\left(z-z_{1}\right)}{z_{1}}+U\left(z_{1}\right) & z_{1}<z<z_{2}, \\
U(z)=\frac{u_{* c}^{2}}{\kappa u_{*} w} \ln \left(\frac{z}{z_{0}}\right) & z_{0}<z<z_{1},
\end{array}
$$

where the no-slip condition at $z_{0}$ and the matching requirement that the velocity is continuous at $z_{1}$ and $z_{2}$ have been imposed. Although 
Equation (2-9) is an explicit solution for the current in this application, a current, $u_{r}$, is specified at a given height above the bottom, $z_{r}$, and $u_{*_{c}}$ is calculated as an inverse problem. This produces a relationship between $u_{w_{c}}$ and $u_{*_{c w}}$.

\subsection{Large roughness}

If the roughness length, $k_{b}\left(=30 z_{0}\right)$, and the wave boundary layer thickness are of the same order of magnitude, then the boundary layer is fully rough turbulent, and it becomes possible for $z_{0}$ to exceed $l_{c w}$. Under these conditions, $z_{0}$ can become greater than $z_{1}$, and the no-slip condition is applied in the range $z_{1}<z_{0}<z_{2}$. The eddy viscosity is constant (Figure $1[b]$ ) near the bed, and the profile throughout the constant stress layer is given by

$$
\begin{aligned}
& K=\kappa u_{* c} z \quad z_{2}<z, \\
& K=\kappa u_{* c w} Z_{1} \quad Z_{1}<Z_{0}<Z<z_{2} .
\end{aligned}
$$

The corresponding kinematic maximum wave stress becomes

$$
u_{* w m}^{2}=\kappa u_{* c w} z_{1} \frac{u_{b}}{l_{c w}} \Gamma_{w s}
$$

where it is understood that $\Gamma_{w s}$ implicitly reflects the change in the solution for the wave shear due to the fact that the eddy viscosity where the no-slip condition is applied is now constant instead of linearly increasing as in Equation(2-5). The solution for $\Gamma_{w s}$ is presented in Appendix A. Similarly, the solution for the current becomes

$$
\begin{array}{rlrl}
U(z) & =\frac{u_{*_{c}}}{\kappa} \ln \left(\frac{z}{z_{2}}\right)+U\left(z_{2}\right) & & z_{2}<z, \\
U(z) & =\frac{u_{*_{c}}^{2}}{\kappa u_{*} w} \frac{\left(z-z_{0}\right)}{z_{1}} & z_{1}<z_{0}<z<z_{2},
\end{array}
$$

where the no-slip condition is now applied above $Z_{1}$.

\subsection{Large roughness with vanishingly small waves}

For the case of the vanishingly small waves, $z_{2} \rightarrow z_{1}$ and $z_{0}$ may even become greater than $Z_{2}$. The eddy viscosity where the no-slip condition is applied then becomes 


$$
K=\kappa u_{* c} Z \quad z_{2}<z_{0}<Z
$$

(Figure $1[\mathrm{c}]$ ), so that

$$
u_{* w m}^{2}=\kappa u_{*} z_{0} \frac{u_{b}}{l_{c w}} \Gamma_{w s}
$$

Note the change in velocity scale from $u_{*_{c w}}$ to $u_{*_{c}}$, which is due to the eddy viscosity above $z_{2}$ being a function of $u_{*_{c}}$ and not $u_{*_{c w}}$. This means that for very small though finite waves, the much greater shear stress associated with the current can still affect the wave. The $\Gamma_{w s}$ solution using the eddy viscosity given by Equation (2-13) is in Appendix A. Substituting Equation (2-13) into Equation (2-4), the current reduces to the classic logarithmic profile,

$$
U(z)=\frac{u_{*_{c}}}{\kappa} \ln \left(\frac{z}{z_{0}}\right) \quad z_{2}<z_{0}<z .
$$

\subsection{Non-dimensionalization of the equations}

An efficient solution algorithm is obtained by recasting the bottom stress equations into a suitable non-dimensional form. Introducing $u_{* w}$ as the velocity scale produces the following non-dimensional parameters that will be useful in formulating the bottom stress solution:

$$
\sigma=\frac{u_{b}}{u_{*_{c} w}}, \quad \epsilon=\frac{u_{*_{c}}}{u_{*_{c w}}}, \quad \mu=\frac{u_{*_{w m}}}{u_{*_{c w}}}
$$

where $\sigma$ is related to a combined wave and current friction factor $\left(\sigma=1 / \sqrt{f_{c w} / 2}\right), \epsilon$ is a measure of the relative contribution from the current to the total stress, and $\mu$ is a measure of the relative contribution from the wave to the total stress. Squaring both sides of Equation (2-2) and rearranging gives

$$
u_{*_{c}}^{4}+2 u_{*_{w m}}^{2} \cos \phi_{c w} u_{*_{c}}^{2}+u_{*_{w m}}^{4}-u_{*_{c} w}^{4}=0
$$

which is quadratic in $u_{*_{c}}^{2}$ with the solution

$$
u_{*_{c}}^{2}=-u_{*_{w m}}^{2} \cos \phi_{c w}+\sqrt{u_{*_{w m}}^{4}\left(\cos ^{2} \phi_{c w}-1\right)+u_{* c w}^{4}}
$$


where the positive addition of the second term is chosen to ensure that $u_{*_{c}}$ is positive. Dividing both sides of Equation (2-18) by $u_{* c w}^{2}$ and substituting $\mu$ and $\epsilon$ from Equation (2-16) yields

$$
\begin{aligned}
\epsilon^{2} & =-\mu^{2} \cos \phi_{c w}+\sqrt{\mu^{4}\left(\cos ^{2} \phi_{c w}-1\right)+1} \\
& =-\mu^{2} \cos \phi_{c w}+\sqrt{1-\mu^{4} \sin ^{2} \phi_{c w}} .
\end{aligned}
$$

The kinematic stress for the wave has three different formulations corresponding to the expressions given by Equations (2-6), (2-11), and (2-14). Dividing both sides of these equations by $u_{c w}^{2}$ and substituting $\mu$ and $\epsilon$ from Equation (2-16) gives

$$
\begin{array}{ll}
\mu^{2}=\kappa \xi_{0} \sigma \Gamma_{w s} & \xi_{0}<\xi_{1}<\xi_{2}, \\
\mu^{2}=\kappa \xi_{1} \sigma \Gamma_{w s} & \xi_{1}<\xi_{0}<\xi_{2}, \\
\mu^{2}=\kappa \xi_{2} \sigma \Gamma_{w s} & \xi_{2}<\xi_{0} .
\end{array}
$$

where $\xi_{1}=z_{1} / l_{c w}$ and $\xi_{2}=z_{2} / l_{c w}$. Similarly, Equations (2-9), (2-12), and (2-15) can be used to formulate three separate expressions for $\sigma$. Rather than outlining the details for all three cases, the non-dimensionalization is illustrated using Equation (2-9). Given a specified current, $u_{r}$, at a height, $z_{r}$, above $z_{2}$, and solving for $\sigma$ yields

$$
\sigma=\frac{\epsilon u_{b}}{\kappa u_{r}}\left[\ln \left(\frac{z_{r}}{z_{2}}\right)+1-\epsilon+\epsilon \ln \left(\frac{z_{1}}{z_{0}}\right)\right]
$$

If the observed current, $u_{r}$, is specified at values of $z_{r}$ that are less than $z_{2}$ or $z_{1}$, but greater than $z_{0}$, three more equations emerge. The resulting solutions for all six formulations are listed in Table 1, along with their appropriate ranges of validity.

The results of the above derivations reveal that $\mu$ and $\sigma$ are dependent on non-dimensional length scales that arise from the eddy viscosity formulation and boundary conditions. These unspecified parameters still must be determined to obtain a closed solution. In general, $\mu$ is a function of $\xi_{0}, \xi_{1}, \sigma$, and $\epsilon$. Only the first two variables remain unspecified, and they will be addressed next. 
Table 1. Expressions for $\sigma$ derived from the current solution discussed in the text. Inequalities signify applicable ranges for a given expression.

\begin{tabular}{|c|c|}
\hline$\sigma=\frac{\epsilon u_{b}}{\kappa u_{r}}\left[\ln \left(\frac{z_{r}}{z_{2}}\right)+1-\epsilon+\epsilon \ln \left(\frac{z_{1}}{z_{0}}\right)\right]$ & $\sigma=\frac{\epsilon^{2} u_{b}}{\kappa u_{r}}\left[\frac{z_{r}}{z_{1}}-1+\ln \left(\frac{z_{1}}{z_{0}}\right)\right] \quad z_{2}>z_{r}>z_{1}>z_{0}$ \\
$z_{r}>z_{2}>z_{1}>z_{0}$ & $(2-25)$ \\
\hline$\sigma=\frac{\epsilon^{2} u_{b}}{\kappa u_{r}} \ln \left(\frac{z_{r}}{z_{0}}\right)$ & $\sigma=\frac{\epsilon u_{b}}{\kappa u_{r}}\left[\ln \left(\frac{z_{r}}{z_{2}}\right)+\epsilon\left(\frac{z_{2}-z_{0}}{z_{1}}\right)\right] \quad(2-27)$ \\
$z_{2}>z_{1}>z_{r}>z_{0}$ & $z_{r}>z_{2}>z_{0}>z_{1}$ \\
\hline$\sigma=\frac{\epsilon^{2} u_{b}}{\kappa u_{r} z_{1}}\left(z_{r}-z_{0}\right)$ & $(2-28)$ \\
$z_{2}>z_{r}>z_{0}>z_{1}$ & $\sigma=\frac{\epsilon u_{b}}{\kappa u_{r}} \ln \left(\frac{z_{r}}{z_{0}}\right)$ \\
\hline
\end{tabular}

The non-dimensional roughness height, $\xi_{0}$, can be written

$$
\xi_{0}=\frac{1}{\kappa R_{*}},
$$

where, analogous to planetary boundary layers (e.g., Grant and Madsen 1986; Wiberg 1995), $R_{*}=u_{* c w} / z_{0} \omega$ is an internal friction Rossby number for combined flows. The parameter $R_{*}$ can be interpreted as the ratio of the nonlinear interaction height to the flow roughness. According to Madsen and Wikramanayake (1991), the dimensional height $z_{1}$ is a function of the wave boundary layer thickness, $z_{1}=\alpha l_{c w}$, where $\alpha$ is a free parameter that represents the fraction of the wave boundary layer in which the eddy viscosity varies linearly with height and is determined experimentally. This defines $\xi_{1}=\alpha$, which gives $\xi_{2}=\alpha / \epsilon$. The parameter $\mu$ is now a function of $R_{*}, \alpha, \sigma$, and $\epsilon$.

Examination of the various solutions presented in Table 1 shows that at a minimum

$$
\sigma=\sigma\left(\frac{u_{b}}{u_{r}}, \frac{z_{1}}{z_{0}}, \frac{z_{r}}{z_{1}}, \epsilon, \alpha\right)
$$

where the explicit functional dependence on $z_{2} / z_{1}$ and $z_{r} / z_{2}$ has been replaced by $z_{2}=z_{1} / \epsilon$. Using the definition for $l_{c w}, z_{1} / z_{0}$ can be written 
$z_{1} / z_{0}=\alpha \kappa R_{*}$. An analogous expression can be defined for $z_{1} / z_{r}$ (i.e., $\left.z_{1} / z_{r}=\alpha \kappa R_{*_{r}}\right)$, where $R_{*_{r}}=u_{*_{c w}} /\left(z_{r} \omega\right)$. The two expressions are related by $R_{*} / R_{* r}=z_{r} / z_{0}$, where $z_{r} / z_{0}$ is an independent external parameter. $R_{*}$ and $\sigma$ are also related since $R_{*} \sigma=u_{b} / \omega z_{0}=A_{b} / z_{0}$, where $A_{b}$ is the bottom wave excursion amplitude. Equation (2-23) is now an implicit function of the external parameters $u_{b} / u_{r}, z_{r} / z_{0}, A_{b} / z_{0}$ and the internal parameters $\alpha$ and $\epsilon$. It can be shown that $\epsilon$ is a function of $A_{b} / z_{0}, \sigma, \alpha$, and $\phi_{c w}$ so that $\sigma$ is a function of the external parameters $u_{b} / u_{r}, z_{r} / z_{0}, A_{b} / z_{0}, \phi_{c w}$, and the internal closure constant $\alpha$. Although the system of coupled equations resulting from this analysis does not produce an algebraic expression for the shear stresses, a closed theoretical solution exists. The nonlinear system therefore can be solved iteratively.

\subsection{Solution algorithm for the three-layer model}

The procedure adopted here is to recast the series of non-dimensional expressions derived above into a root-finding algorithm for $\sigma$. Applying the pure current $\left(u_{b}=0\right)$ or pure wave $\left(u_{c w}=u_{w m}\right)$ limit shows that $\sigma$ is bounded by $0 \leq \sigma \leq u_{b} / u_{* w m}$. The solution for the pure wave limit is obtained by setting $\mu=1$ and can be computed independently of the combined stress solution. Depending on the root-finding algorithm, at least one initial guess for $\sigma$ is needed to start the iteration. For combined flows, the bisection method is used (Atkinson 1989) since the root is guaranteed to lie between the pure wave and pure current limits. The next step is to determine $\mu$, which has a functional dependence that can be described by

$$
\mu=\mu\left(\frac{A_{b}}{z_{0}}, \alpha, \sigma, \epsilon\right) .
$$

The first two parameters are given, the third is assigned an initial value that lies between the universal limits, and the last parameter is unknown. Recalling that $\epsilon$ is related to $\mu$ and the external parameter $\phi_{c w}$ through Equation (2-19), an internally consistent value can be computed by recasting the coupled Equations (2-19) and (2-20) into a root-finding algorithm for $\epsilon$ similar to that used to determine $\sigma$. Again, the bisection method is chosen since $\epsilon$ is bounded by universal limits $(0 \leq \epsilon \leq 1)$. Once $\mu$ and $\epsilon$ have been computed, $z_{1} / z_{0}$ is determined by $z_{1} / z_{0}=\alpha \kappa R_{*}=\alpha \kappa A_{b} / z_{0} / \sigma$, and $z_{1} / z_{r}$ is determined by $z_{1} / z_{r}=\alpha \kappa R_{*_{r}}=\alpha \kappa A_{b} / z_{r} / \sigma$. The parameters $z_{2} / z_{r}$ and $z_{2} / z_{0}$ are related to $z_{1} / z_{r}$ and $z_{1} / z_{0}$ through $\epsilon$, which is now known.

Given the above estimates for these non-dimensional length scales, along 
with $u_{b} / u_{r}$, which is an external parameter, a new value for $\sigma$ is computed from the equations listed in Table 1 . The relative difference between the new and old value of sigma is checked to see if it is below some prescribed tolerance. If it is not, then the process is repeated until $\sigma$ converges (the tolerance threshold is reached).

\subsection{Simplification for $\mu$}

The solution procedure described above reveals that a nested iteration scheme is required, in which an inner loop is first initiated to produce internally consistent estimates of $\mu$ and $\epsilon$, and then an outer loop is executed to solve for $\sigma$. These iterations represent the most computationally expensive operations in the stress solution. If the inner loop can be removed from the solution procedure, then the total number of computations will be reduced, increasing the speed of convergence.

An examination of the governing equation for the wave (Styles and Glenn 2000) indicates that the velocity scale $\left(u_{*_{c}}\right)$ for the stress term when $z>z_{2}$ is identical to the formulation above the wave boundary layer derived by GM. Using scaling arguments for the governing equation for the wave, GM demonstrated that as long as $u_{w_{c}}$ was on the order of the wave velocity or less, then the stress term for the wave outside the wave boundary layer could be neglected. For the case here, which considers pure currents as a possible limit, their assumption may not apply when the current is much stronger than the wave. Under these circumstances, the wave shear and associated wave stress for $z>z_{2}$ are relatively weak so that the wave solution in the outer region is well described by the linear theory, except possibly under very rough conditions (Styles and Glenn 2000). If the stress term for the wave is neglected above $z_{2}$, then the solution for $\mu$ becomes independent of $\epsilon$ and therefore $z_{2}$. This eliminates the inner iteration loop required to produce an internally consistent value for $\mu$ and $\epsilon$ and accelerates the speed of convergence without appreciably altering the results of the stress model based on a three-layer eddy viscosity for $\mu$.

Appendix B presents a derivation of $\Gamma_{w s}$ and $\mu$ based on a simpler, continuous two-layer eddy viscosity (Figure 1 [d], [e]) in which the stress term for the wave above $z_{2}$ is neglected. The solution procedure follows that described in Section 2.5 except that $\mu$ no longer depends on $\epsilon$. Instead, $\epsilon$ is computed explicitly through Equation (2-19). 


\section{Model Results}

To illustrate the properties of the stress model, results are presented based on the simplified solution for $\mu$ discussed in Section 2.6. The input parameters consist of the external variables $A_{b} / z_{0}, z_{r} / z_{0}, u_{b} / u_{r}$, and $\phi_{c w}$ and the internal closure constant $\alpha$.

Because the stress model is designed for a broad range of input wave and current conditions that may be produced by a shelf circulation model, the parameter ranges for $A_{b} / z_{0}$ and $z_{r} / z_{0}$ are $10^{-} 3 \leq A_{b} / z_{0} \leq 10^{6}$ and $1.01 \leq z_{r} / z_{0} \leq 10^{6}$, respectively. The lower limit is chosen to represent a maximum relative roughness for the wave $\left(z_{0} / A_{b}\right)$ of $10^{3}$. The upper limit is chosen to represent a $100 \mathrm{~cm}$ current height $\left(z_{r}\right)$ or excursion amplitude $\left(A_{b}\right)$ over a ripple-free bed with a minimum grain diameter roughness of $\sim 10^{-3} \mathrm{~cm}$ (10 microns $\left.[\mu \mathrm{m}]\right)$. Other model parameters have been fixed with values of $\alpha=1, \phi_{c w}=0$, and $u_{b} / u_{r}=1$. The latter is chosen so that the wave and current outside the wave boundary layer are approximately the same order of magnitude. Past expressions for $\alpha$ have ranged between approximately 0.15 and 2 (Glenn 1983; Madsen and Wikramanayake 1991; Lynch et al. 1997). Therefore, an intermediate value (1.0) is chosen to illustrate the model characteristics.

\subsection{Fundamental model characteristics}

Figure 2 depicts selected internal model parameters identified in the text as a function of the independent external parameters $z_{r} / z_{0}$ and $A_{b} / z_{0}$. Individual model parameters show varying degrees of sensitivity to $z_{r} / z_{0}$ and $A_{b} / z_{0}$, especially for extreme values. The first two parameters, $\mu=u_{*_{w m}} / u_{*_{c w}}$ and $\epsilon=u_{*_{c}} / u_{*_{c w}}$, illustrate the dynamic features of the stress model since they define the relative proportions of the wave and current shear velocities to the total. The pure wave or pure current limits are easily interpreted graphically as $\mu \rightarrow 1$ and $\epsilon \rightarrow 0$ for pure waves, and $\mu \rightarrow 0$ and $\epsilon \rightarrow 1$ for pure currents. As $z_{r} / z_{0} \rightarrow 1$ for a fixed $u_{r}$, the current shear becomes very large. This produces a larger stress for the current $(\epsilon \rightarrow 1$ ) that will dominate over the wave in the combined flow. As this ratio increases, the current shear begins to decrease, with an associated reduction in $\epsilon$. Eventually, $z_{r} / z_{0}$ will become so large that the turbulent stresses associated with the current (for constant $u_{r}$ ) must vanish and $\epsilon=0$. In this limit, the solution becomes that of a pure wave $(\mu \rightarrow 1)$. The rate at which the pure wave limit is approached is also a 
function of $A_{b} / z_{0}$. For constant $u_{b}$, decreases in $A_{b} / z_{0}$ lead to greater frictional drag for the wave and an associated increase in bottom stress. This behavior is apparent in the first two plots, as the pure wave limit proceeds more rapidly as a function of $z_{r} / z_{0}$ for smaller $A_{b} / z_{0}$.

Figure 2. Selected internal model parameters as a function of $Z_{r} / z_{0}$ and $A_{b} / z_{0}$. Definitions of the internal variables are provided in the text. Values for $A_{b} / z_{0}$ range from $10^{-3}$ to $10^{6}$ in decadal increments.

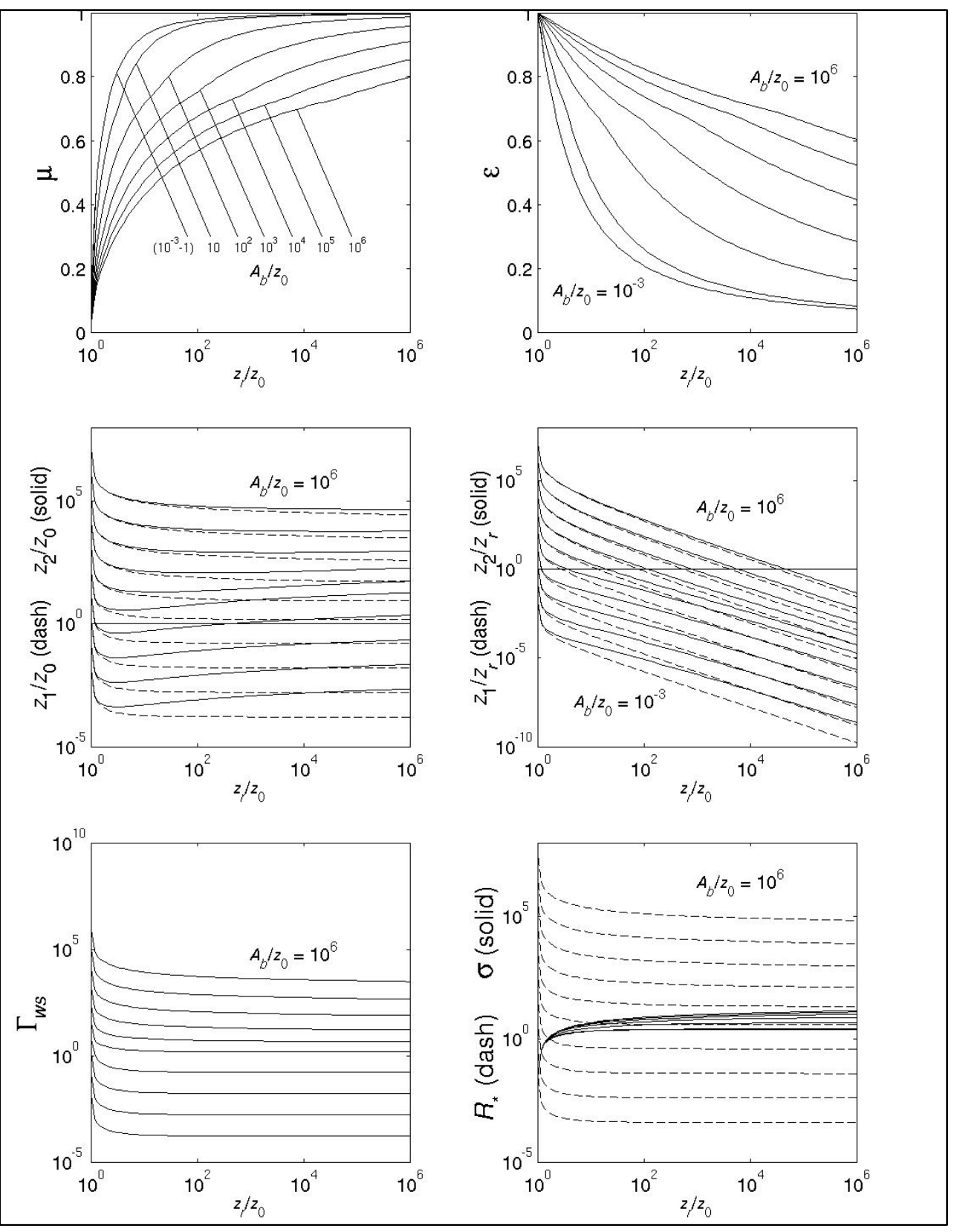


Another notable feature is that both $\mu$ and $\epsilon$ become independent of $A_{b} / z_{0}$ when this ratio is less than 1 . This can be understood by examining the non-dimensional length scales $z_{1} / z_{0}$ and $z_{2} / z_{0}$, and the parameter $\sigma$ as a function of $z_{r} / z_{0}$, when $10^{-} 3 \leq A_{b} / z_{0} \leq 10^{-1}$. Figure 2 reveals that $z_{1} / z_{0}$ and $z_{2} / z_{0}$ are always less than one in this range so that the eddy viscosity at the bed becomes constant and $\mu^{2}=\kappa \sqrt{\epsilon_{1}} \sigma$ (Appendix B). Substituting $\sigma$ from Equation (2-29) into the above expression for $\mu$ gives

$$
\mu^{2}=\sqrt{\xi_{1}} \epsilon \frac{u_{b}}{u_{r}} \ln \left(\frac{z_{r}}{z_{0}}\right) \quad z_{r}>z_{0}>z_{2}>z_{1},
$$

which is independent of $A_{b} / z_{0}$. The parameter $\epsilon$ is related to $\mu$ through Equation (2-19) and is also independent of $A_{b} / z_{0}$.

The second group of parameters, $z_{1} / z_{0}, z_{2} / z_{0}, z_{1} / z_{r}$, and $z_{2} / z_{r}$, represents the length scales of the flow. All four parameters exhibit a strong dependence on $A_{b} / z_{0}$, but only $z_{1} / z_{r}$ and $z_{2} / z_{r}$ are dependent of $z_{r} / z_{0}$ when this ratio becomes very large. An important consideration for modeling applications is that all four parameters are well behaved for the broad range of $A_{b} / z_{0}$ and $z_{r} / z_{0}$ used here. The only exception occurs when $z_{r} / z_{0}=1\left(u_{r}=0\right)$, which is a degenerate case.

$\Gamma_{w s}$ is sensitive to smaller values of $z_{r} / z_{0}$ and to $A_{b} / z_{0}$ as long as $z_{1} / z_{0}>1$. When $z_{1} / z_{0}<1$, the eddy viscosity that defines the maximum wave shear is constant, and the resulting equation for $\Gamma_{w s}$, which is derived in Appendix $\mathrm{B}$, is independent of $A_{b} / z_{0}$. The final two parameters, $R_{*}$ and $\sigma$, are both sensitive to smaller values of $z_{r} / z_{0}$, and $R_{*}$ is sensitive to $A_{b} / z_{0}$ for all values. The parameter $\sigma$ is not very sensitive to the very large changes in either $z_{r} / z_{0}$ or $A_{b} / z_{0}$. This demonstrates the advantage of selecting $\sigma$ as the function best suited for a root-finding algorithm to close the stress solution.

The stress model also depends on $u_{b} / u_{r}$, which is a measure of the relative strength of the wave to the current. Figure 3 shows $\mu, \epsilon$, and $\sigma$ for the same conditions illustrated in Figure 2 but with $u_{b} / u_{r}=10$ (large wave) and 0.1 (small wave). The general trends are the same as the $u_{b} / u_{r}=1$ case, with the exception that for larger $u_{b} / u_{r}$ the solution approaches that of a pure wave much faster as a function of $z_{r} / z_{0}$, and for $u_{b} / u_{r}=0.1$ the change is more gradual. Since a larger $u_{b} / u_{r}$ signifies a stronger ambient wave, it is expected that the solution should converge to the pure wave 
limit much more rapidly as $z_{r} / z_{0}$ increases. The opposite is true for a relatively large current $\left(u_{b} / u_{r}=0.1\right)$. The parameter $\sigma$ also is not as sensitive to $A_{b} / z_{0}$ when the current is much stronger than the wave.

Figure 3. Similar to Figure 2 but showing only $\mu, \epsilon$, and $\sigma$. Left column is for $u_{b} / u_{r}=10$ (large waves), and right column is for $u_{b} / u_{r}=0.1$ (large currents).
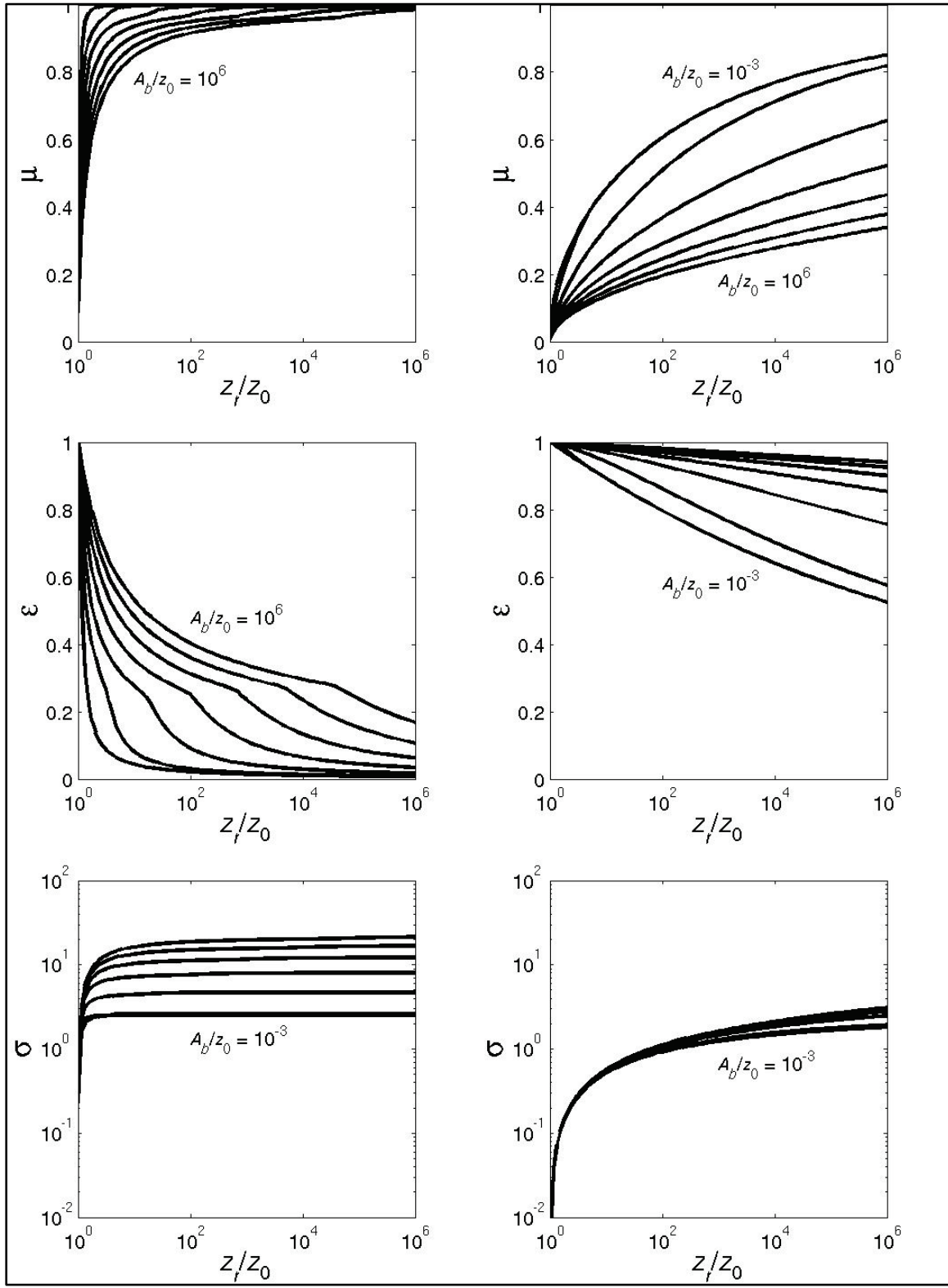

\subsection{Sensitivity to the direction between the wave and current}

Equation (2-19) expresses a closed relationship between $\epsilon$ and $\mu$ given the external parameter $\phi_{c w}$. A plot of $\epsilon$ as a function of $\mu$ for several values of $\phi_{c w}$ is shown in Figure 4. For small $\mu, \epsilon$ is not very sensitive to $\mu$ or $\phi_{c w}$. As 
$\mu$ becomes larger, and the shear stress associated with the wave becomes significant, $\epsilon$ becomes much more sensitive to $\phi_{c w}$. This sensitivity can be described mathematically, since Equation (2-19) reduces to

$$
\epsilon^{2}=1-\mu^{2}
$$

for co-directional flow $\left(\phi_{c w}=0\right)$ and to

$$
\epsilon^{2}=\sqrt{1-u^{4}}
$$

for orthogonal flow $\left(\phi_{c w}=\pi / 2\right)$. The larger exponent for $\mu$ in Equation (3-3) tends to make $\epsilon$ larger when the wave and current are at right angles. Physically, this means that for a fixed maximum wave stress vector in the presence of a current, the magnitude of the time-averaged shear stress must continually increase as $\phi_{c w}$ goes from o to $\pi / 2$, if the magnitude of the maximum total stress vector is to remain constant. The direction of the maximum total stress vector will of course change as the time-averaged shear stress vector rotates toward $\pi / 2$. During storms, the wave and current vectors near the coast are generally at a high angle (closer to orthogonal than co-directional) and both are relatively strong. If topographic steering or an evolving current (e.g., tides) produces local regions where $\phi_{c w}$ becomes small, an associated increase in the magnitude of the total shear stress may occur. Several studies have examined combined flows in which the angle between the wave and current is 90 degrees (Fernando et al. 2011; Madsen et al. 2008; Musumeci et al. 2006). There have been fewer observational studies to characterize the stress within the wave boundary layer for arbitrary wave and current vectors. Some studies indicate that a first-order effect is a reduction in the bottom roughness for the current as $\phi_{c w}$ increases (Sorenson et al. 1995; Styles 1998; Madsen et al. 2008; Styles and Bryant 2016). 
Figure 4. Sensitivity of the parameters $\epsilon$ and $\mu$ to the direction between the wave and the current.

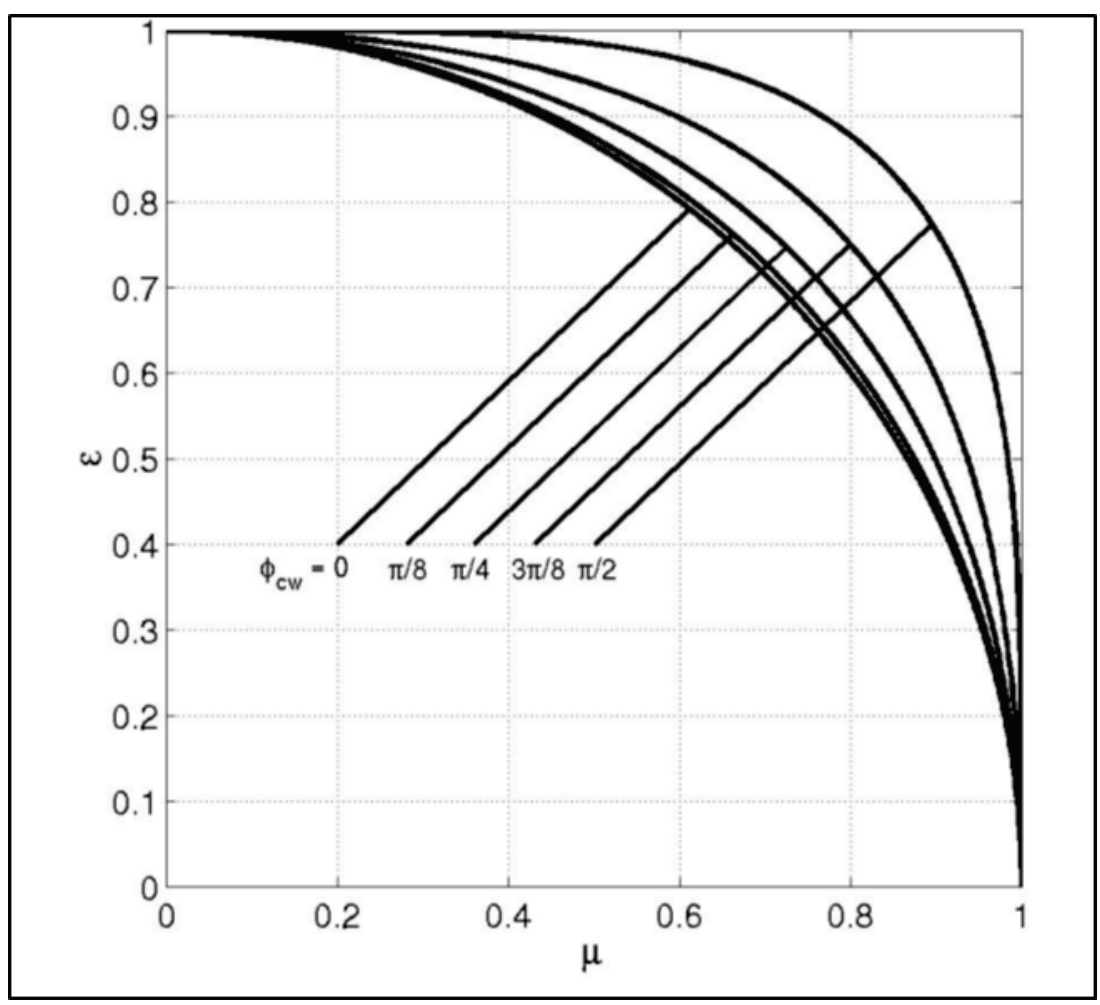

\subsection{Bottom stress sensitivity to the eddy viscosity profile}

The solution presented above neglects the stress term in the governing equation for the wave above $z_{2}$ but retains it for the current. This was justified based on the assumption that $u_{*_{c}}$ was on the order of the wave velocity or less (GM). Styles and Glenn (2000) also have argued that the details of the eddy viscosity outside the wave boundary layer are not important in determining the bed stress except possibly for very rough beds. Both the Styles and Glenn (2000) three-layer eddy viscosity depicted in Figure 1 (a, b, c) and the simplified, two-layer continuous eddy viscosity for the wave depicted in Figure 1 (d, e) are identical as $z \rightarrow z_{0}$ but diverge above $z_{2}$. Another eddy viscosity profile that has been used extensively in the past is the linearly increasing, discontinuous form originally proposed by GM (Figure 1 [f]). Since all three formulations are different above the wave boundary layer but the same below $z_{1}$, the present stress model can be used to examine how the details of the eddy viscosity profile outside the wave boundary layer affect bed stress estimates.

A stress model based on the GM eddy viscosity does not include the $z_{1}$ or $z_{2}$ terms. Instead, GM prescribe the height of the wave boundary layer, $\delta_{c w}$, 
which is also formulated as a constant, $n$, times $l_{c w}\left(\delta_{c w}=n l_{c w}\right)$. For this comparison, $n$ is set equal to 2, which is the typical value used in applications (Glenn and Grant 1987; Madsen et al. 1993; Madsen 1994; Keen and Glenn 1994). To highlight the differences between the three eddy viscosity formulations, the ranges of the input variables are reduced to $10^{-1} \leq A_{b} / z_{0} \leq 10^{3}$ and $1.01 \leq z_{r} / z_{0} \leq 10^{3}$. Other parameters are set with values of $\phi_{c w}=0, u_{b} / u_{r}=1$ and $\alpha=1$. To illustrate the dynamical properties of the three modeling approaches, the comparison focuses on the parameters $\mu, \epsilon$, and $\sigma$, which are depicted in Figure 5. For $A_{b} / z_{0} \geq 100$, all three models produce approximately the same result, and therefore, the solution is not sensitive to the form of the eddy viscosity in the outer wave boundary layer and above. This point is argued by Styles and Glenn (2000), who claim that the stratification correction also introduces arbitrary changes to the eddy viscosity and therefore can be neglected in the wave stress solution.

Figure 5. Comparison of $\mu, \epsilon$, and $\sigma$ for the GM (thin solid), Styles and Glenn (2000) (dash), and two-layer continuous (thick solid) eddy viscosity profiles. $A_{b} / z_{0}$ ranges from $10^{-1}$ to $10^{3}$ in decadal increments.

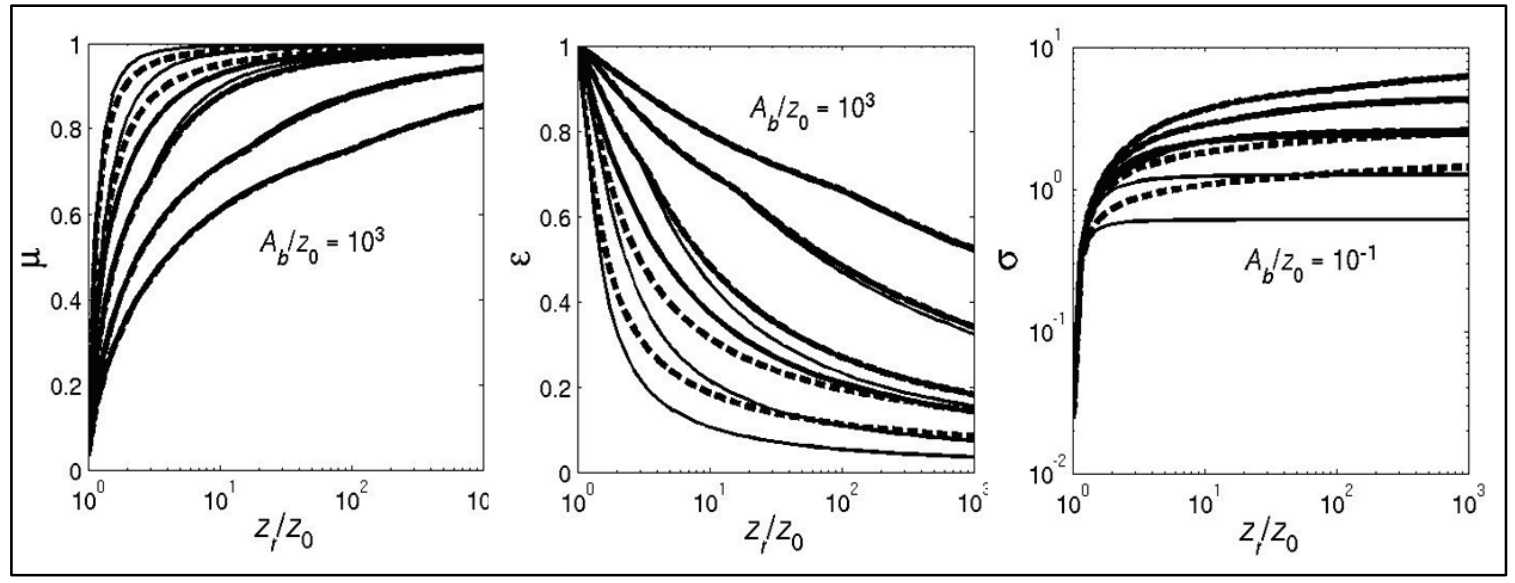

For a larger relative roughness, the three solutions begin to diverge. This is most apparent for the two-layer continuous eddy viscosity, which was shown to produce an upper bound on $\mu$ and a lower bound on $\epsilon$ and $\sigma$ when $z_{1} / z_{0}<1$. The GM and Styles and Glenn (2000) eddy viscosity profiles for the wave are not constant in the outer portion of the constant stress layer so that $\sigma, \mu$, and $\epsilon$ remain functions of $A_{b} / z_{0}$ when the relative roughness is very large.

In the limit of a pure current $\left(z_{r} / z_{0} \rightarrow 1\right)$, both $\mu$ and $\epsilon$ based on the stress models derived using the GM and Styles and Glenn (2000) eddy 
viscosities converge. This limit was mentioned above as a possible case when $u_{*_{c}}$ might be important in the governing equation for the wave outside the wave boundary layer. Since the result based on the Styles and Glenn (2000) eddy viscosity profile includes the stress term above $z_{2}$ and the GM eddy viscosity does not, the importance of retaining the stress term for this case appears minimal. The fact that the three models produce dissimilar results for a large relative roughness $\left(A_{b} / z_{0} \lesssim 10\right)$ suggests that careful consideration of the parameterization of the eddy viscosity for a rough bed is important.

\subsection{Evaluation of $\alpha$}

The above analysis has revealed that the model is most sensitive to the eddy viscosity profile for very rough beds $\left(k_{b} / A_{b} \gtrsim 1.0\right)$. On storm-dominated sandy continental shelves, the roughest beds are speculated to be associated with the presence of relic ripples, which can have maximum ripple heights that exceed $10 \mathrm{~cm}$ (Traykovski et al. 1999). The amount of time that relic ripples dominate the roughness signature on sandy continental shelves is a function of the state of ripple degradation after storms (Nelson and Voulgaris 2014). Assuming that relic ripples persist for some time after storm events, it is possible to estimate the average amount of time that relic ripples may be present. Studies of storm-forced transport on the New Jersey shelf (Styles 1998) have indicated that the average storm, as defined by the time that the shear stress based on skin friction exceeds the minimum for the initiation of sediment motion, lasts approximately 24 hours and that approximately 10 such storms occur annually. Assuming that biological activity sufficiently degrades ripples within a week or two after a storm (Traykovski et al. 1999) gives an annual maximum relic ripple period of 2 to 5 months. This can be a significant amount of time and indicates that BBLMs must be designed to accommodate a variety of roughness conditions that are likely to occur on wave-dominated continental shelves.

Model sensitivity to the form of the eddy viscosity profile can be investigated by modulating the parameter $\alpha$. If $\alpha$ is relatively large, then the eddy viscosity in the vicinity of $z_{0}$ increases linearly with height. This is the same as the GM model deep within the wave boundary layer. For intermediate values of $\alpha$, the eddy viscosity is identical to Madsen and Wikramanayake (1991) and Styles and Glenn (2000). If $\alpha$ is very small, then the eddy viscosity in the vicinity of $z_{0}$ is constant, and it is similar to vertically uniform profiles that have been suggested for very rough beds (Nielsen 1992; Sleath 1991). Modifying $\alpha$ in the present model effectively 
reproduces the eddy viscosity profiles discussed above, which were shown to produce different results when $k_{b} / A_{b}$ was greater than approximately 1 .

For a given set of external wave, current, and roughness conditions, the boundary shear stress becomes sensitive only to the value of $\alpha$. In laboratory flumes, all of these parameters can easily be prescribed or measured independently of a combined flow model with the exception of the bottom roughness. This is because the precise mathematical formulation depends on the size and shape of the bedforms present, which means that $k_{b}$ varies as a function of the experimental conditions and is not universal in form. Therefore, the experimental setting must conform as closely as possible to the actual environmental conditions to which the calibration results apply. In this case, the experimental conditions must include roughness elements that simulate the approximate shape of wavegenerated ripples and more importantly they must return a consistent roughness value based on several independent methods of determination.

The data sets used to evaluate $\alpha$ for combined flows are obtained from Mathisen and Madsen (1996a, b) (hereinafter referred to as MM). MM conducted detailed experiments of co-directional wave and current flows in a laboratory flume. To ensure rough turbulent conditions, they modified the bed of their flume with triangular shaped bars that were scaled to simulate the geometry of two-dimensional (2-D) wave-generated ripples. In nearly all of their experiments, $k_{b} / A_{b}>1$, which is ideal for evaluating the present stress model for rough conditions. MM reported all necessary input data to drive the model including bottom roughness height and the wave friction factor, which was determined by measuring the decay in wave height over the length of the flume and relating that to dissipation due to bottom friction. Noting that $\mu / \sigma=\sqrt{f_{w} / 2}$, friction factor curves can be generated and compared to their measurements. Setting $k_{b}$ equal to the roughness determined for pure currents (MM 1996a, Table 2) and using the measured water particle amplitudes, orbital velocities and mean currents, a family of friction factor curves as a function of $\alpha$ is generated. The current roughness is chosen since MM's results demonstrated that $k_{b}$ was nearly the same for waves in the presence and absence of currents, and currents in the presence and absence of waves. Also, their current roughness estimates are independent of the GM combined wave and current model, whereas this model is used to determine the roughness for all their cases with waves. As noted above, increasing $\alpha$ leads to an eddy viscosity profile that is very similar to the GM formulation. The decision to 
use the roughness length for pure currents ensures that the present method to determine $\alpha$ is not inherently dependent on the GM model (through the bottom roughness), which may bias the results to favor larger values of $\alpha$. To quantify the comparison between the model and data, there is an adoption of the relative error defined by

$$
\ln (e)=\left[\frac{1}{N} \sum_{i=1}^{N}\left(\ln \left(Y_{i}\right)-\ln \left(\bar{Y}_{i}\right)\right)^{2}\right]^{\frac{1}{2}}
$$

where $Y_{i}$ is the measured data point, $\bar{Y}_{i}$ is the corresponding model estimate, and $N$ is the number of data points (Wikramanayake and Madsen 1991). The friction factor curve that minimizes $e$ identifies the optimum $\alpha$.

Using values that range from 0.15 to 2 (Glenn 1983; Madsen and Wikramanayake 1991; Lynch et al. 1997; Styles 1998), the lowest error ( $e=$ 1.3) is obtained when $\alpha$ is set equal to 0.75 . The corresponding modeled wave boundary layer thickness, as determined by $\delta_{c w}=n l_{c w}(n=2)$, is a factor of 3 too low when compared to the observed height derived from wave and current profile measurements in the flume. MM also underestimate the height of the wave boundary layer using the GM model. MM attribute the enhanced boundary layer thickness to the increased bed roughness associated with their fixed artificial roughness elements. Relic ripples may play a role similar to artificial roughness elements, and therefore, may produce an enhanced boundary layer thickness relative to smoother flow conditions. Although the main purpose here is to describe an algorithm to compute the total bed stress, the resulting wave and current stress components are integral components of suspended sediment concentration and velocity profile models (i.e., Smith 1975; Wiberg and Smith 1983; GM; Glenn and Grant 1987). A model designed to predict current and suspended sediment concentration profiles should be able to reproduce accurately both the friction factor (stress) and the wave boundary layer thickness.

The fact that the model tends to underestimate the thickness of the wave boundary layer, yet accurately predicts the wave friction factor, suggests that it is the internal length scales, which define the height and thickness of the various regions in the boundary layer, as opposed to the velocity scales, which define the shear stresses, that should be reexamined. The 
present and GM model have adjustable internal length scales $z_{1}$ and $\delta_{c w}$, respectively. It is hypothesized that for very rough conditions $\left(k_{b} / A_{b} \gtrsim 1\right)$, the constants multiplying $l_{c w}$ in the definition of $z_{1}$ and $\delta_{c w}$ are now functions of the relative roughness. A very simple approximation that incorporates explicitly the relative roughness in the definition of $z_{1}$ but reverts to the existing formulation in the limit $k_{b} / A_{b} \rightarrow 0$ is to modify $z_{1}$ as $z_{1}=\alpha l_{c w}\left(a_{0}+a_{1} k_{b} / A_{b}+a_{2}\left(k_{b} / A_{b}\right)^{2}+\ldots\right)$. Since $k_{b} / A_{b}$ is expected to become a leading order term only for very rough beds, the series can be truncated to first order to give $z_{1}=\alpha l_{c w}\left(1+\beta k_{b} / A_{b}\right)$. A similar expression is proposed for $\delta_{c w}: \delta_{c w}=n l_{c w}\left(1+\beta k_{b} / A_{b}\right)$. For smooth to moderately rough turbulent conditions $\left(k_{b} / A_{b} \lesssim 0.1\right)$, the GM model has been shown to accurately predict the shear stress and apparent roughness with $n=2$ (e.g., Grant et al. 1984; Drake and Cacchione 1992; Drake et al. 1992). This leaves two undetermined parameters ( $\alpha$ and $\beta$ ) that must be calibrated from data. Optimal values are found by choosing the combination that minimizes the relative difference for both the friction factor and the wave boundary layer thickness. Using a range of values for $\beta$ similar to those chosen for $\alpha$, the lowest error $(e=1.2)$ is obtained when $\alpha=0.3$ and $\beta=$ 0.7. The results for combined flows are presented in Figure 6 . The model compares well with the measured combined wave and current friction factors, and the average wave boundary layer thickness of $6.2 \mathrm{~cm}$ determined from the model ( $\alpha=0.3$ ) compares well with the measured value of $6 \mathrm{~cm}$.

MM also conducted experiments for pure waves (MM 1996a, Table 1). The roughness elements were the same as in the combined flow and pure current cases. Given that MM demonstrated similar roughness for waves in the presence and absence of currents permits an additional opportunity to refine the closure parameters in the case of pure waves. Setting the roughness height equal to the average obtained by MM for the pure current case, and using their experimental input wave parameters, friction factor curves are generated from the model and compared to their data. The results are shown in Figure 7. The lowest error $(e=1.2)$ for the friction factor was obtained with $\alpha=0.3$ and $\beta=0.8$. The average wave boundary layer thickness determined from the model was $6.0 \mathrm{~cm}$. When $\beta$ was set equal to 0.7, the lowest error for the friction factor still occurred with $\alpha=$ o.3, but the modeled wave boundary layer thickness had a mean of $5.4 \mathrm{~cm}$. In both the combined and pure wave case, a consistent result emerges in which the calibration coefficients maintain similar values. It must be emphasized that the suggested values for the closure constants are only 
valid as long as they are applied to the stress model presented above (MM). Other wave/current bottom boundary layer models that include similar modifications must be calibrated before they are used in applications.

Figure 6. Combined wave and current model calibration results for the closure parameters $\alpha$.

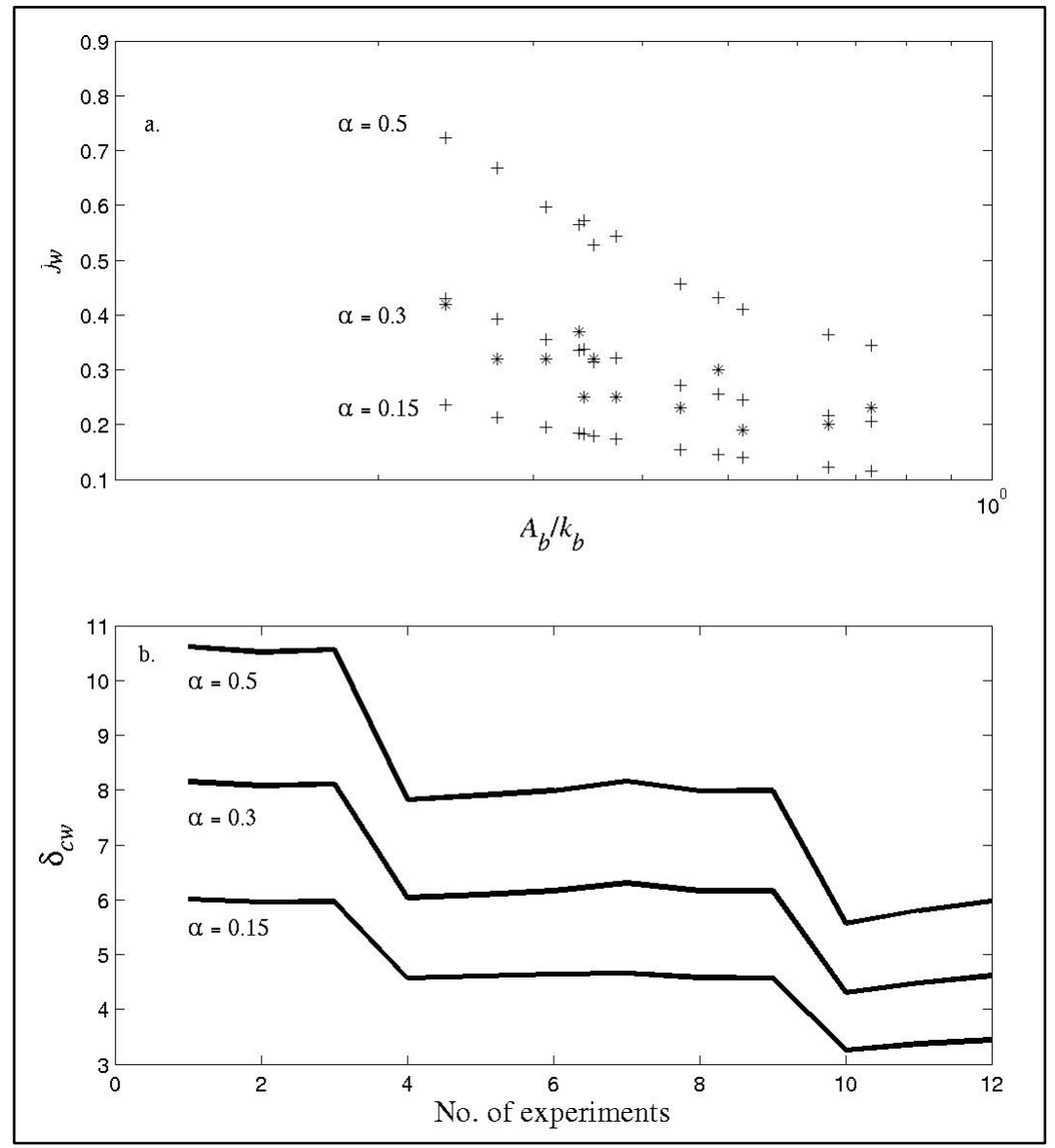


Figure 7. Pure wave model calibration results for the closure parameters $\alpha$ and $\beta$.

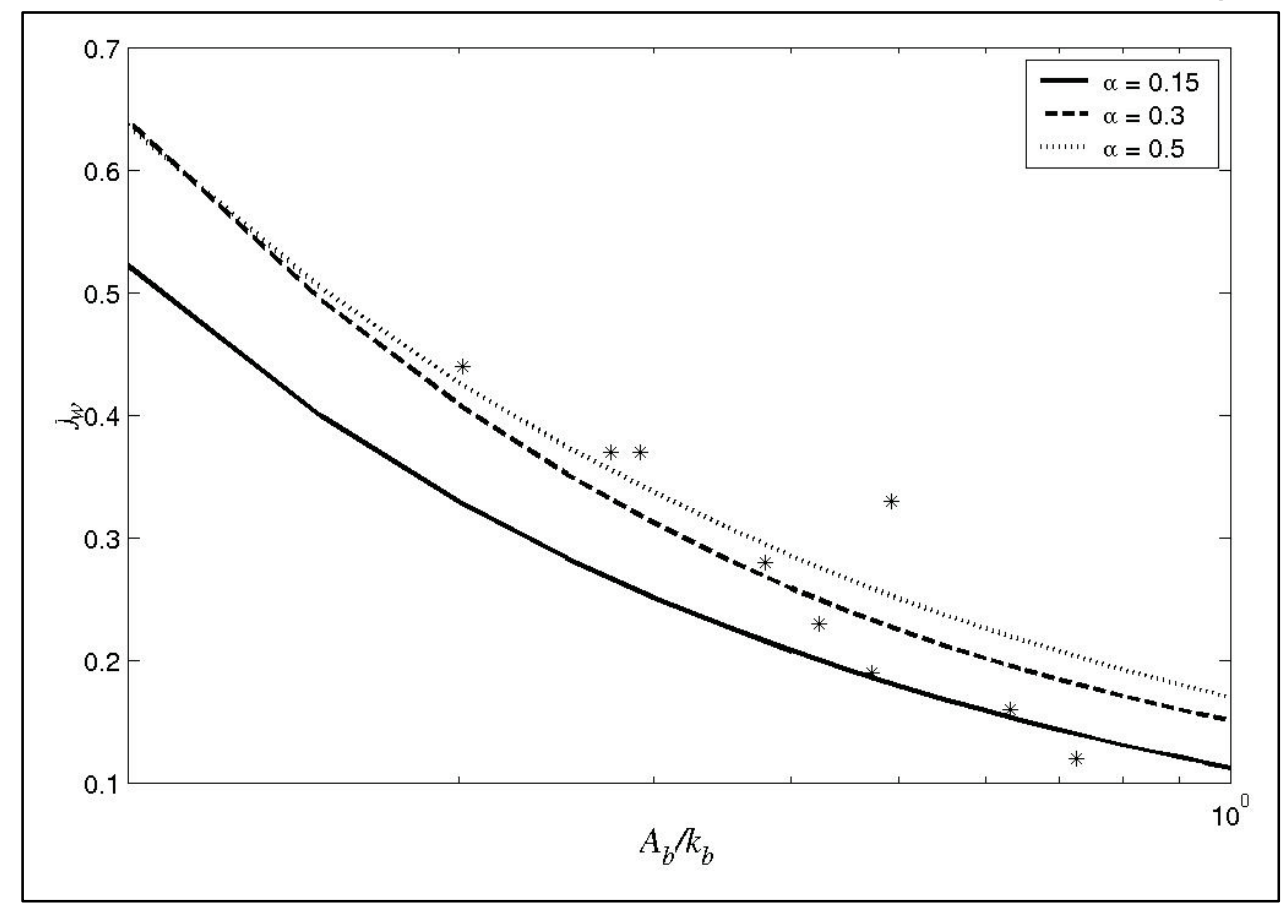

The modifications presented above are not without a theoretical or empirical basis. The first-order correction to $z_{1}$ leads to an eddy viscosity profile that is similar in functional form to expressions developed by Nielsen (1992) and Sleath (1991). For rough oscillatory flow very near the bed, Nielsen (1992) proposed the eddy viscosity $K=0.004 A_{b}^{3 / 2} k_{b}^{1 / 2} \omega$ for $A_{b} / k_{b}<16$. Similarly, Sleath (1991) suggested $K=0.0025 A_{b} k_{b} \omega$ in the range $1<A_{b} / k_{b}<120$. Both expressions share a common functional dependence, namely the nonlinear product $A_{b}^{c} k_{b}^{d} \omega$, with the constraint that $c+\mathrm{d}=2$. The calibration results presented above are formulated in terms of an eddy viscosity in the transition layer $\left(z_{1}<z<z_{2}\right)$ that is written as $K=\kappa u_{* c w} z_{1}$, with $z_{1}=\alpha l_{c w}\left(1+\beta k_{b} / A_{b}\right)$. Like the Nielsen (1992) and Sleath (1991) results, this modification leads to an eddy viscosity profile that is also an implicit nonlinear function of the product $A_{b} k_{b} \omega$. To illustrate, a pure wave in which the roughness is large enough so that $z_{1} / z_{0}<1$ is considered. In this case, the eddy viscosity becomes $K=\kappa u_{* w} \alpha l_{* w m}\left(1+\beta k_{b} / A_{b}\right)$. Noting that $u_{*_{w m}} / u_{b}=\sqrt{f_{w} / 2}$ along with the definition of $l_{w m}\left(=\kappa u_{w_{w m}} / \omega\right)$, the eddy viscosity can be written as $K=\alpha \kappa^{2} f_{w} / 2 A_{b} \omega\left(A_{b}+\beta k_{b}\right)$. Expanding this expression, for example, for constant $f_{w}$, gives an eddy viscosity that is proportional to $A_{b} k_{b} \omega$, which has the same functional form as Sleath (1991). Depending on the definition of $f_{w}$, other nonlinear expressions emerge. As an example, Kajiura (1968) derived a friction factor of the form $f_{w}=0.35\left(k_{b} / A_{b}\right)^{2 / 3}$. Substitution of this expression gives $K=0.175 \alpha \kappa^{2}\left(A_{b}^{4 / 3} k_{b}^{2 / 3} \omega+\beta A_{b}^{1 / 3} k_{b}^{5 / 3} \omega\right)$. Although this 
functional form is different from the results of Nielsen (1992) or Sleath (1991), all three formulations share a common nonlinear dependence on the same external parameters $k_{b}, A_{b}$, and $\omega$. The present modification, in that the explicit dependence on $k_{b} / A_{b}$ vanishes in the limit of smoother bed conditions, is somewhat more general than the Nielsen (1992) and Sleath (1991) models that apply only for $k_{b} / A_{b}$ greater than approximately 0.008 .

\subsection{Speed of convergence tests}

An advantage of the solution algorithm described in this report is that the nested iteration scheme used in Styles and Glenn (2000) and the family of Grant, Madsen, and Glenn models (GM; Glenn and Grant 1987) can be avoided. Since the iterative root-finding algorithm is the most computationally expensive step in the solution procedure, it is of great advantage if the number of times this operation must be executed can be substantially reduced. In fact, Keen and Glenn (1994) spent considerable effort to optimize the initial guess for the friction factor and other variables to speed the convergence in their streamlined version of the GM BBLM.

To illustrate the computational advantage of the present approach over the Styles and Glenn (2000) model, which uses the same eddy viscosity profile but still uses a nested iteration scheme, results of a speed of convergence test are presented. Because the Styles and Glenn (2000) model is restricted to a much narrower range of wave, current, and roughness environments, the input parameters represent only a small subset of the full capabilities of the stress model presented here. The values of the input parameters, normalized run-time, and total number of iterations are listed in Table 2. Each row represents 10,000 independent model runs with identical input and initial conditions. The run-time was recorded for each run and normalized to produce the numbers listed in Table 2. The numbers in parentheses under the Styles and Glenn (2000) model denote the maximum number of iterations required for the friction factor convergence. The other set of numbers denote the number of iterations required for $u_{*_{c}}$ convergence in the Styles and Glenn (2000) model and $\sigma$ in the present model. The Styles and Glenn (2000) model uses the secant method while the present model uses a variation of Brent's root-finding algorithm (Atkinson 1989), in which the iterations are performed using the bisection method but convergence is checked using the secant method after only a few iterations. A tolerance limit of $10^{-4}$, or a $0.01 \%$ relative error between the previous and present iteration, is designated to established convergence. 
Table 2. Speed of convergence tests comparing the present method with the unstratified version of the Styles and Glenn (2000) wave and current BBLM. For all model runs, $\phi_{c w}=0$ and $Z_{r}=100 \mathrm{~cm}$. The first three rows are for strong waves and currents (SS), the middle three rows are for strong waves and weak currents (SW), and the last three rows are for weak waves and strong currents (WS). The last two columns list normalized run-time (RT) and total number of iterations (N) for each method. The Styles and Glenn (2000) model uses a nested iteration scheme. The numbers in parentheses indicate the maximum number of iterations for the inner loop, which usually occurred during the first or second iteration of the outer loop.

\begin{tabular}{|c|c|c|c|c|c|c|c|c|}
\hline & $u_{b}$ & $A_{b}$ & $u_{r}$ & $k_{b}$ & \multicolumn{2}{|c|}{ Present } & \multicolumn{2}{|c|}{ Styles and Glenn } \\
\hline & $(\mathrm{cm} / \mathrm{s})$ & $(\mathrm{cm})$ & $(\mathrm{cm} / \mathrm{s})$ & $(\mathrm{cm})$ & $\mathrm{RT}$ & $\mathrm{N}$ & $\mathrm{RT}$ & $\mathrm{N}$ \\
\hline SS1 & 50 & 100 & 20 & 1.0 & 1 & 7 & 3.6 & $5(10)$ \\
\hline SS2 & 50 & 100 & 20 & 10 & 1 & 7 & 2.8 & $5(9)$ \\
\hline SS3 & 50 & 100 & 20 & 100 & 1 & 5 & 2.9 & $5(7)$ \\
\hline & & & & & & & & \\
\hline SW1 & 50 & 100 & 1 & 1.0 & 1 & 8 & 4.4 & $7(8)$ \\
\hline SW2 & 50 & 100 & 1 & 10 & 1 & 9 & 2.6 & $7(7)$ \\
\hline SW3 & 50 & 100 & 1 & 100 & 1 & 9 & 2 & $6(6)$ \\
\hline & & & & & & & & \\
\hline WS1 & 1 & 2 & 50 & 1.0 & 1 & 4 & 21 & $3(37)$ \\
\hline WS2 & 1 & 2 & 50 & 10 & 1 & 6 & 7.3 & $5(17)$ \\
\hline WS3 & 1 & 2 & 50 & 100 & 1 & 5 & 7 & $5(18)$ \\
\hline
\end{tabular}

The results indicate that in all cases, convergence proceeds with fewer iterations, is at least twice as fast, and in some cases an order of magnitude faster, thus illustrating the greater efficiency of the bottom stress algorithm presented here. A similar speed of convergence comparison was performed between the present and the GM model, which can also be formulated without a nested iteration scheme (Grant and Madsen 1986). The results were similar except for the smoother conditions ( $k_{b}=1$ or 10 ), in which case the present formulation usually converged $20 \%$ to $30 \%$ faster. Since the present model uses a more physically realistic eddy viscosity profile, has a correction to produce accurate estimates of the wave boundary layer thickness for very rough beds, and is more efficient for smoother conditions than the Grant and Madsen (1986) model, it is recommended for applications in which estimates of the near-bed flow and suspended sediment concentration profiles are desired. 


\section{Summary}

The authors have presented a fairly robust algorithm to compute the enhanced wave and current boundary shear stress components for an unstratified bottom boundary layer. The stress model was designed for a broad range of wave and current flows and was formulated without the need to introduce fictitious currents in the constant stress layer to obtain closure. Instead, the governing equations for the wave and current were reviewed and used to identify important velocity and length scales that could characterize the flow for a broad range of wave and current conditions, including the limiting case of pure waves or pure currents. Systematic non-dimensionalization of the governing equations revealed three important internal parameters: $\epsilon=u_{*_{c}} / u_{*_{c w}}, \mu=u_{*_{w m}} / u_{*_{c w}}$ and $\sigma=u_{b} / u_{* c w}$. It was demonstrated that interpreting the functional dependence of $\epsilon, \mu$, and $\sigma$ graphically helped to illustrate model stability and to distinguish the effects of different turbulence closure methods (i.e., different eddy viscosity formulations). The bed shear stress was most sensitive to the form of the eddy viscosity in the outer wave boundary layer and above for rough flow conditions.

For very rough conditions, available combined wave and current data were utilized to refine estimates of the empirical constant $\alpha$. To resolve the discrepancy between past formulations that produced accurate estimates of the friction factor but underestimated the thickness of the wave boundary layer, the scale heights $Z_{1}$ and $\delta_{c w}$ were modified to include an explicit dependence on the relative roughness. This introduced an additional closure constant, $\beta$, that was determined experimentally to be approximately 0.7 for combined flows and 0.8 for pure waves. Further analysis of combined wave and current flows over very rough beds in natural flows is needed before a definitive value can be prescribed to model the constant stress portion of the bottom boundary layer. Until then, it is suggested that $\alpha=0.3$ and $\beta=0.7$ for applications of the stress model presented here.

Speed of convergence tests revealed that the present model converged in fewer total iterations and much faster than the Styles and Glenn (2000) BBLM, which used the same eddy viscosity profile. Faster convergence was attributed to the more efficient solution method, which avoided the nested iteration scheme used by Styles and Glenn (2000) and the family of Grant, Madsen, and Glenn models. 


\section{References}

Atkinson, K. E. 1989. An Introduction to Numerical Analysis. New Jersey: John Wiley \& Sons, Inc.

Benetazzo, A., A. Bergamasco, D. Bonaldo, F. M. Falcieri, M. Sclavo, L. Langone, and S. Carniel. 2014. "Response of the Adriatic Sea to an Intense Cold Air Outbreak: Dense Water Dynamics and Wave-Induced Transport." Progress in Ocean. 128:115-138.

Cacchione, D. A., and D. E. Drake. 1982. "Measurements of Storm-Generated Bottom Stresses on the Continental Shelf." J. Geophys. Res. 87:1,952-1,960.

Cooper, C., and J. D. Thompson. 1989. "Hurricane-Generated Currents on the Outer Continental Shelf. 1: Model Formulation and Verification.” J. Geophys. Res. 94:12,513-12,539.

Davies, A. G., and C. Villaret. 1997. "Oscillatory Flow over Rippled Beds: Boundary Layer Structure and Wave-Induced Eulerian Drift.” Gravity Waves in Water of Finite Depth, Vol 10. Southampton, UK: Computational Mechanics Publications. 215-254.

Davies, A. M., and J. Lawrence. 1994. "Examining the Influence of Wind and Wind Wave Turbulence on Tidal Currents, Using a Three-Dimensional Hydrodynamic Model Including Wave-Current Interaction.” J. Phys. Oceanogr. 24:2,441-2,460.

Drake, D. E., and D. A. Cacchione. 1992. "Wave-Current Interaction in the Bottom Boundary Layer during Storm and Non-Storm Conditions: Observations and Model Predictions." Cont. Shelf Res. 12:1,331-1,352.

Drake, D. E., D. A. Cacchione, and W. D. Grant. 1992. "Shear Stress and Bed Roughness Estimates for Combined Wave and Current Flows over a Rippled Bed.” $J$. Geophys. Res. 97:2,319-2,326.

Fernando, P. C., J. Guo, and P. Lin. 2011. "Wave-Current Interaction at an Angle 1: Experiment.” J. Hydraulic Res. 49:424-436.

Glenn, S. M. 1983. A Continental Shelf Bottom Boundary Layer Model: The Effects of Waves, Currents, and a Movable Bed. Sc.D. thesis, Mass. Inst. of Technol., Cambridge.

Glenn, S. M., and W. D. Grant. 1987. "A Suspended Sediment Correction for Combined Wave and Current Flows.” J. Geophys. Res. 92:8,244-8,246.

Grant, W. D., and O. S. Madsen. 1979. "Combined Wave and Current Interaction with a Rough Bottom.” J. Geophys. Res. 89(84):1,797-1,808.

Grant, W. D., and O. S. Madsen. 1986. "The Continental-Shelf Bottom Boundary Layer." Ann. Rev. Fluid Mech. 18:265-305.

Grant, W. D., A. J. Williams, III, and S. M. Glenn. 1984. "Bottom Stress Estimates and Their Prediction on the Northern California Continental Shelf During CODE-1: The importance of wave-current interaction.” J. Phys. Oceanogr. 14:506-527.

Kajiura, K. 1968. "A Model of the Bottom Boundary Layer in Water Waves.” Bulletin of the Earthquake Research Institute 46:75-123. 
Keen, T. R., and S. M. Glenn. 1994. "A Coupled Hydrodynamic-Bottom Boundary Layer Model of Ekman Flow on Stratified Continental Shelves.” J. Phys. Oceanogr. 24:1,733-1,749.

Keen, T. R., and S. M. Glenn. 1995. "A Coupled Hydrodynamic-Bottom Boundary Layer Model of Storm and Tidal Flow in the Middle Atlantic Bight of North America." J. Phys. Oceanogr. 25:391-406.

Keen, T. R., and S. M. Glenn. 1998. "Factors Influencing Model Skill for Hindcasting Shallow Water Currents during Hurricane Andrew.” J. Atmos. Oceanic Technol. 15:221-236.

Keen, T. R., and R. L. Slingerland. 1993a. "A Numerical Study of Sediment Transport and Event Bed Genesis during Tropical Storm Delia.” J. Geophys. Res. 98:4,775-4,791.

Keen, T. R., and R. L. Slingerland. 1993b. "Four Storm-Event Beds and the Tropical Cyclones That Produced Them: A Numerical Hindcast.” Journal of Sedimenatry Research 63:218-232.

Lynch, J. F., J. D. Irish, T. F. Gross, P. L. Wiberg, A. E. Newhall, P. A. Traykovski, and J. D. Warren. 1997. "Acoustic Measurements of the Spatial and Temporal Structure of the Near-Bottom Boundary Layer in the 1990-1991 STRESS Experiment." Cont. Shelf Res. 17:1,271-1,295.

Madsen, O. S., and P. N. Wikramanayake. 1991. Simple Models for Turbulent WaveCurrent Bottom Boundary Layer Flow. U.S. Army Corps of Engineers, Coastal Engineering Research Center, Contract report DRP-91-1, 150 pp.

Madsen, O. S. 1994. "Spectral Wave-Current Bottom Boundary Layer Flows.” In Proceedings of the $24^{\text {th }}$ Coastal Engineering Conference, Kobe, 384-398.

Madsen, O. S., L. D. Wright, J. D. Boon, and T. A. Chisholm. 1993. "Wind Stress, Bed Roughness and Sediment Suspension on the Inner Shelf during an Extreme Storm Event.” Cont. Shelf Res. 13:1,303-1,324.

Madsen, O. S., K. Kularatne, H. Cheong. 2008. "Experiments on Bottom Roughness Experienced by Currents Perpendicular to Waves." In Proc. $31^{\text {st }}$ International Conference on Coastal Engineering, Hamburg, 845-853.

Mathisen, P. P., and O. S. Madsen. 1996a. "Waves and Currents over a Fixed Rippled Bed 1. Bottom Roughness Experienced by Waves in the Presence and Absence of Currents.” J. Geophys. Res. 101:16,533-16,542.

Mathisen, P. P., and O. S. Madsen. 1996b. "Waves and Currents over a Fixed Rippled Bed 2. Bottom and Apparent Roughness Experienced by Currents in the Presence of Waves.” J. Geophys. Res. 101:16,543-16,550.

Musumeci, R., L. Cavallaro, E. Foti, P. Scandura, and P. Blondeaux. 2006. "Waves Plus Currents Crossing at a Right Angle: Experimental Investigation.” Journal of Geophysical Research: Oceans 111.

Nelson, T., and G. Voulgaris. 2014. "Temporal and Spatial Evolution of Wave-Induced Rippled Geometry: Regular Versus Irregular Ripples.” J. Geophys., Res. 119:664688. 
Nielsen, P. 1981. "Dynamics and Geometry of Wave-Generated Ripples." J. Geophys. Res. $86: 6,467-6,472$.

Nielsen, P. 1992. "Coastal Bottom Boundary Layers and Sediment Transport." World Scientific 324.

Signell, R. P., R. C. Beardsley, H. C. Graber, and A. Capotondi. 1990. "Effect of WaveCurrent Interaction on Wind-Driven Circulation in Narrow, Shallow Embayments.” Journal of Geophysical Research C: Oceans 95:9671-9678.

Sleath, J. F. A. 1991. "Velocities and Shear Stresses in Wave-Current Flows." J. Geophys. Res. 96:15,237-15,244.

Smith, J. D. 1975. "Modeling of Sediment Transport on Continental Shelves.” The Sea, ed. E. D. Goldberg, I. N. McCave, J. J. O’Brien, J. H. Steele. 6, Interscience 538-577.

Sorenson, K. S., O. S. Madsen, and L. D. Wright. 1995. "Evidence of DirectionalDependence of Moveable Bottom Roughness in Inner Shelf Waters." Eos Trans. $A G U 76$ (1995):46.

Spaulding, M. L., and T. Isaji. 1985. "Design Flow Conditions near Bottom, Phase II, Coupling of a Continental Shelf Hydrodynamics Model to a Bottom Boundary Layer Model." Vol. 1, Theoretical Development and Application. Final Rep. to Pipeline Res. Comm., Project PR-169-1186, Amer. Gas Assoc.

Styles, R. 1998. A Continental Shelf Bottom Boundary Layer Model: Development, Calibration and Applications to Sediment Transport in the Middle Atlantic Bight. Ph.D. thesis, Rutgers, The State Univ. of New Jersey, New Brunswick.

Styles, R., and S. M. Glenn. 200o. "Modeling Stratified Wave and Current Bottom Boundary Layers on the Continental Shelf." J. Geophys. Res. 105:24,119-14,139.

Styles, R., and D. Bryant. 2016. Combined Wave and Current Bottom Boundary Layers: A Review. ERDC-CHL-SR-16-1. Vicksburg, MS: U.S. Army Engineer Research and Development Center.

Traykovski, P., A. E. Hay, J. D. Irish, and J. F. Lynch. 1999. "Geometry, Migration, and Evolution of Wave Orbital Ripples at LEO-15.” J. Geophys. Res. 104:1,505-1,524.

Warner, J. C., C. R. Sherwood, R. P. Signell, C. K. Harris, and H. G. Arango. 2008. "Development of a Three-Dimensional, Regional, Coupled Wave, Current, and Sediment-Transport Model." Computers \& Geosciences 34:1,284-1,306.

Wiberg, P., and J. D. Smith. 1983. "A Comparison of Field Data and Theoretical Models for Wave-Current Interactions at the Bed on the Continental Shelf." Cont. Shelf Res. 2:126-136.

Wiberg, P. 1995. "A Theoretical Investigation of Boundary Layer Flow and Bottom Shear Stress for Smooth, Transitional, and Rough Flow under Waves.” J. Geophys. Res. 100:22,667-22,679.

Yuan, J., and O. S. Madsen. 2015. "Experimental and Theoretical Study of Wave-Current Turbulent Boundary Layers.” J. Fluid Mech. 765:480-523. 


\section{Appendix A: Derivation of Non-dimensional Wave Shear under Strong Waves}

Presented here is a derivation of $\Gamma_{w s}$ and $\mu$ for the eddy viscosity in Equation (2-5) when $\xi_{0}$ is greater than $\xi_{1}$ or $\xi_{2}$. Invoking the usual linear and boundary layer approximations, Styles and Glenn (2000) present the governing equation for the wave within the wave boundary layer for the three-layer eddy viscosity as

$$
\begin{array}{rlrl}
i W-\frac{\partial}{\partial \xi} \xi \epsilon \frac{\partial W}{\partial \xi} & =0 & \xi_{2}<\xi \\
i W-\frac{\partial}{\partial \xi} \xi_{1} \frac{\partial W}{\partial \xi}=0 & \xi_{1}<\xi<\xi_{2} \\
i W-\frac{\partial}{\partial \xi} \xi \frac{\partial W}{\partial \xi}=0 & \xi_{0}<\xi<\xi_{1}
\end{array}
$$

where $W=u_{w}-u_{b}$. A simple harmonic motion $\left(e^{i \omega t}\right)$ so that the time dependence is separable from the $\xi$ dependence is assumed. With the appropriate boundary and matching conditions (Styles and Glenn 2000), the solution for the modulus of the wave is written

$$
\begin{array}{ll}
u_{w}=\mid u_{b}+G(\operatorname{Ker} 2 \sqrt{\xi / \epsilon}+i \operatorname{Kei} 2 \sqrt{\xi / \epsilon} \mid & \xi_{2}<\xi, \\
u_{w}=\left|u_{b}+C e^{m \xi}+D e^{-m \xi}\right| & \xi_{1}<\xi<\xi_{2}, \\
u_{w}=\left|u_{b}+A(\operatorname{Ber} 2 \sqrt{\xi}+i \operatorname{Bei} 2 \sqrt{\xi})\right| & \xi 0<\xi<\xi_{1}
\end{array}
$$

where $A, B, C, D$, and $G$ are complex constants, Ber, Bei, Ker, and Kei are zero-order Kelvin functions, and $m=\sqrt{i / \xi_{1}}$. For convenience, the $e^{i \omega t}$ term is dispensed with since in this example the modulus of a complex number $(f+i g)$ times $e^{i \omega t}$ is simply the modulus of $f+i g$. The values of the constants can be found in Madsen and Wikramanayake (1991) and reflect the specific condition that $\xi_{0}<\xi_{1}<\xi_{2}$. Based on the governing equation of Equation (A-5) and solution of Equation(A-6), it is possible to extend the wave solution to include cases when $\xi_{1}<\xi_{0}<\xi_{2}$ and $\xi_{1}<\xi_{2}<\xi_{0}$. 
Case 1: $\xi_{1}<\xi_{0}<\xi_{2}$

Using the eddy viscosity profile given in Equation (2-10), the governing equation for $W$ can be written as

$$
\begin{array}{rlrl}
i W & =\frac{\partial}{\partial \xi} \xi \epsilon \frac{\partial W}{\partial \xi}=0 & \xi_{2}<\xi, \\
i W=\frac{\partial}{\partial \xi} \xi_{1} \frac{\partial W}{\partial \xi}=0 & \xi_{0}<\xi<\xi_{2} .
\end{array}
$$

Invoking the no-slip condition at the bed and given that the solution smoothly approaches the potential flow result at the top of the boundary layer, the corresponding solution for the wave modulus is

$$
\begin{array}{ll}
u_{w}=\mid u_{b}+G^{\prime}(\operatorname{Ker} 2 \sqrt{\xi / \epsilon}+i \operatorname{Kei} 2 \sqrt{\xi / \epsilon} \mid & \xi_{2}<\xi, \\
u_{w}=\left|u_{b}+C^{\prime} e^{m \xi}+D^{\prime} e^{-m \xi}\right| & \xi_{0}<\xi<\xi_{2}
\end{array}
$$

where

$$
C^{\prime}=\frac{-u_{b} L}{P_{0} L+M_{0} N}, \quad D^{\prime}=\frac{-u_{b} N}{P_{0} L+M_{0} N}
$$

and

$$
G^{\prime}=2\left[\frac{C^{\prime} P_{2}+D^{\prime} M_{2}}{K_{2}}+p\left(\frac{C^{\prime} P_{2}-D^{\prime} M_{2}}{K_{2}^{(1)}}\right)\right]
$$


The terms in Equations (A-9) and (A-10) are defined as follows:

$$
\begin{gathered}
M_{0}=e^{-m \xi_{0}}, \quad M_{2}=e^{-m \xi_{2}}, \\
P_{0}=e^{m \xi_{0}}, \quad P_{2}=e^{m \xi_{2}}, \\
K_{2}=\operatorname{Ker} 2 \sqrt{\xi_{2} / \epsilon}+i \operatorname{Kei} 2 \sqrt{\xi_{2} / \epsilon}, \\
K_{2}^{(1)}=\left.\frac{\partial}{\partial \xi}\left(\operatorname{Ker} 2 \sqrt{\xi_{2} / \epsilon}+i \operatorname{Kei} 2 \sqrt{\xi_{2} / \epsilon}\right)\right|_{\xi=\xi_{2}}, \\
L=M_{2}\left(m K_{2}+K_{2}^{(1)}\right), \quad N=P_{2}\left(m K_{2}+K_{2}^{(1)}\right) .
\end{gathered}
$$

Substituting the wave solution into Equation (2-7), $\Gamma_{w s}$ becomes

$$
\Gamma_{w s}=|m| \frac{M_{0} N-P_{0} L}{M_{0} N+P_{0} L}||
$$

and

$$
\mu^{2}=\kappa \xi_{1} \sigma \Gamma_{w s} .
$$

Case 2: $\xi_{1}<\xi_{2}<\xi_{0}$

For this case, Equation (2-13) defines the eddy viscosity (Figure 1 [c]) so that the solution for the wave modulus in the vicinity of $\xi_{0}$ becomes

$$
u_{w}=u_{b}\left|1-\frac{\operatorname{Ker} 2 \sqrt{\xi / \epsilon}+i \operatorname{Kei} 2 \sqrt{\xi / \epsilon}}{\operatorname{Ker} 2 \sqrt{\xi_{0} / \epsilon}+i \operatorname{Kei} 2 \sqrt{\xi_{0} / \epsilon}}\right| \quad \xi_{2}<\xi_{0} .
$$

Inserting Equation (A-14) into Equation (2-7) gives

$$
\Gamma_{w s}=\left|\frac{-\frac{\partial}{\partial \xi}\left(\operatorname{Ker} 2 \sqrt{\xi_{0} / \epsilon}+i \operatorname{Kei} 2 \sqrt{\xi_{0} / \epsilon}\right)}{\operatorname{Ker} 2 \sqrt{\xi_{0} / \epsilon}+i \operatorname{Kei} 2 \sqrt{\xi_{0} / \epsilon}}\right|
$$

and

$$
\mu^{2}=\kappa \xi_{0} \epsilon \sigma \Gamma_{w s} .
$$




\section{Appendix B - Derivation of Non-dimensional Wave Shear under Weak Waves}

Presented here is the solution for $\Gamma_{w s}$ and $\mu$ while neglecting the stress term in the governing equation for the wave above $\xi_{2}$.

\section{Case 1: Two-layer eddy viscosity $\left(z_{0}<Z_{1}\right)$}

For the two-layer eddy viscosity presented in Figure 1 (d), the governing equation for $W$ is similar in form to the lower two layers in Equation (A-5). Applying the appropriate boundary and matching conditions, the modulus of the wave solution becomes

$$
\begin{aligned}
& u_{w}=\left|u_{b}+D^{\prime \prime} e^{-m \xi}\right| \quad \xi_{1}<\xi, \\
& u_{w}=\mid u_{b}+A^{\prime}(\operatorname{Ber} 2 \sqrt{\xi}+i B e i 2 \sqrt{\xi}) \\
& +B^{\prime}(\operatorname{Ker} 2 \sqrt{\xi}+i \operatorname{Kei} 2 \sqrt{\xi}) \mid \quad \xi_{0}<\xi<\xi_{1},
\end{aligned}
$$

where

$$
A^{\prime}=\frac{-u_{b} N^{\prime}}{B_{0} N^{\prime}-K_{0} L^{\prime}}, \quad B^{\prime}=\frac{-u_{b} L}{K_{0} L^{\prime}-B_{0} N^{\prime}}
$$

and

$$
D^{\prime \prime}=\frac{A^{\prime}\left(B_{1}+B_{1}^{(1)}\right)+B^{\prime}\left(K_{1}+K_{1}^{(1)}\right)}{M_{1}(1-m)} .
$$


The terms in Equations (B-2) and (B-3) are defined as follows:

$$
\begin{gathered}
K_{0}=\operatorname{Ker} 2 \sqrt{\xi_{0}}+i \operatorname{Kei} 2 \sqrt{\xi_{0}}, \quad B_{0}=\operatorname{Ber} 2 \sqrt{\xi_{0}}+i \operatorname{Bei} 2 \sqrt{\xi_{0}}, \\
K_{1}=\operatorname{Ker} 2 \sqrt{\xi_{1}}+i \operatorname{Kei2} \sqrt{\xi_{1}}, \quad B_{1}=\operatorname{Ber} 2 \sqrt{\xi_{1}}+i \operatorname{Bei} 2 \sqrt{\xi_{1}}, \\
K_{1}^{(1)}=\left.\frac{\partial}{\partial \xi}\left(\operatorname{Ker} 2 \sqrt{\xi_{1}}+i \operatorname{Kei} 2 \sqrt{\xi_{1}}\right)\right|_{\xi=\xi_{1}}, \\
B_{1}^{(1)}=\left.\frac{\partial}{\partial \xi}\left(\operatorname{Ber} 2 \sqrt{\xi_{1}}+i \operatorname{Bei} 2 \sqrt{\xi_{1}}\right)\right|_{\xi=\xi_{1}}, \\
L^{\prime}=m B_{1}+B_{1}^{(1)}, \quad N^{\prime}=m K_{1}+K_{1}^{(1)}, \\
M^{\prime}=e^{-m \xi_{1}} .
\end{gathered}
$$

Substituting the modulus into Equation (2-7) yields

$$
\Gamma_{w s}=\mid-\left[\frac{N^{\prime} B_{0}^{(1)}}{B_{0} N^{\prime}-K_{0} L^{\prime}}+\frac{L^{\prime} K^{(1)_{0}}}{K_{0} L^{\prime}-B_{0} N^{\prime}} \mid,\right.
$$

and

$$
\mu^{2}=\kappa \xi_{0} \sigma \Gamma_{w s}
$$

Case 2: Two-layer eddy viscosity $\left(z_{0}>z_{1}\right)$

If $\xi_{0}$ is greater than $\xi_{1}$, the wave modulus becomes

$$
u_{w}=\left|u_{b}\left[1-e^{-m\left(\xi-\xi_{0}\right)}\right]\right| \quad \xi_{1}<\xi_{0}<\xi
$$

With the aid of Equation (2-7), $\Gamma_{w s}$ takes on the simple form

$$
\Gamma_{w s}=|m|
$$

and

$$
\mu^{2}=\kappa \xi_{1} \sigma \Gamma_{w s}=\kappa \sqrt{\xi_{1}} \sigma
$$




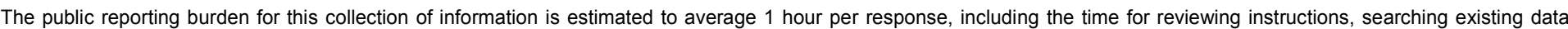

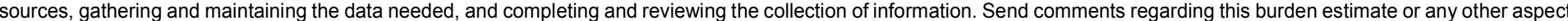

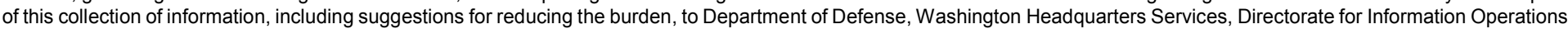

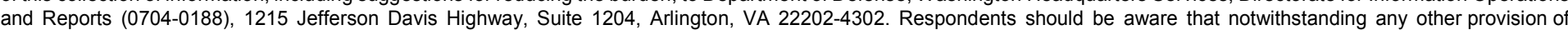
law, no person shall be subject to any penalty for failing to comply with a collection of information if it does not display a currently valid OMB control number. PLEASE DO NOT RETURN YOUR FORM TO THE ABOVE ADDRESS.

\begin{tabular}{l|l|l}
\hline $\begin{array}{l}\text { 1. REPORT DATE } \\
\text { June } 2017\end{array}$ & $\begin{array}{l}\text { 2. REPORT TYPE } \\
\text { Final Report }\end{array}$ & 3. DATES COVERED (FrOm - To) \\
\hline
\end{tabular}

\section{TITLE AND SUBTITLE}

5a. CONTRACT NUMBER

An Optimized Combined Wave and Current Bottom Boundary Layer Model for

Arbitrary Bed Roughness

5b. GRANT NUMBER

5c. PROGRAM ELEMENT NUMBER

6. AUTHOR(S)

Richard Styles, Scott M. Glenn, and Mitchell E. Brown 5d. PROJECT NUMBER

462583

5e. TASK NUMBER

5f. WORK UNIT NUMBER

\section{PERFORMING ORGANIZATION NAME(S) AND ADDRESS(ES) (see reverse)}

9. SPONSORING/MONITORING AGENCY NAME(S) AND ADDRESS(ES)

U.S. Army Corps of Engineers

Washington, DC 20314-1000

\section{SPONSOR/MONITOR'S REPORT}

NUMBER(S) REPORT NUMBER

ERDC/CHL TR-17-11

10. SPONSOR/MONITOR'S ACRONYM(S)

HQUSACE
PERFORMING ORGANIZATION

\section{DISTRIBUTION/AVAILABILITY STATEMENT}

Approved for public release; distribution is unlimited.

\section{SUPPLEMENTARY NOTES}

\section{ABSTRACT}

A robust method for computing the bed shear stress in unstratified combined wave and current flows is presented. The present approach follows from existing theories describing the nonlinear wave and current interaction in the benthic boundary layer but is designed for arbitrary wave, current, and roughness conditions, including the limiting case of pure waves or pure currents. The stress model is intended as a stand-alone application or for coupling to three-dimensional shelf circulation models, where a broad range of flow conditions are encountered. High-quality data for combined flows and pure waves are used with the present stress formulation to better refine empirical model closure constants in the fully rough turbulent regime. Introducing a first-order correction to the definition of the wave boundary layer thickness produces accurate estimates of both the measured friction factor and wave boundary layer height. A speed of convergence test indicates that the present model is more efficient than previous models that use the same turbulent closure scheme. This is primarily due to an improved solution algorithm that avoids the nested iterations common to established combined wave and current bottom boundary layer models.

\section{SUBJECT TERMS}

Boundary layer, Coastal sediments, Littoral drift, Mathematical models, Ocean bottom, Ocean circulation, Ocean currents, Ocean waves

\section{SECURITY CLASSIFICATION OF:}

\begin{tabular}{l|l|l|}
\hline a. REPORT & b. ABSTRACT & c. THIS PAGE \\
Unclassified & Unclassified & Unclassified \\
\hline
\end{tabular}

17. LIMITATION OF
ABSTRACT
SAR

\section{NUMBER OF PAGES}

43 19a. NAME OF RESPONSIBLE PERSON Mitchell E. Brown

19b. TELEPHONE NUMBER (Include area code) 601-634-4036 
Coastal and Hydraulics Laboratory

U.S. Army Engineer Research and Development Center 3909 Halls Ferry Road

Vicksburg, MS 39180-6199

Institute of Marine and Coastal Sciences

Rutgers, The State University of New Jersey

57 US Highway 1

New Brunswick, NJ 08901-8554 